\title{
Review \\ Plant-Based Synthesis of Gold Nanoparticles and Theranostic Applications: A Review
}

\author{
Uday M. Muddapur ${ }^{1, *(\mathbb{D})}$, Sultan Alshehri ${ }^{2} \mathbb{D}$, Mohammed M. Ghoneim ${ }^{3}\left(\mathbb{D}\right.$, Mater H. Mahnashi ${ }^{4}(\mathbb{D}$, \\ Mohammed Abdulrahman Alshahrani ${ }^{5}$, Aejaz Abdullatif Khan ${ }^{6}$, S. M. Shakeel Iqubal ${ }^{6, *(D)}$, Amal Bahafi ${ }^{7}$, \\ Sunil S. More ${ }^{8}$, Ibrahim Ahmed Shaikh ${ }^{9}$ (D), Basheerahmed Abdulaziz Mannasaheb ${ }^{3}$, Noordin Othman ${ }^{10,11}$, \\ Muazzam Sheriff Maqbul ${ }^{12}$ and Mohammad Zaki Ahmad ${ }^{13}$ (D)
}

\section{check for}

updates

Citation: Muddapur, U.M.; Alshehri,

S.; Ghoneim, M.M.; Mahnashi, M.H.; Alshahrani, M.A.; Khan, A.A.; Iqubal, S.M.S.; Bahafi, A.; More, S.S.; Shaikh, I.A.; et al. Plant-Based Synthesis of Gold Nanoparticles and Theranostic Applications: A Review. Molecules 2022, 27, 1391. https://doi.org/ 10.3390/molecules 27041391

Academic Editor: Jessica

Rosenholm

Received: 12 January 2022

Accepted: 16 February 2022

Published: 18 February 2022

Publisher's Note: MDPI stays neutral with regard to jurisdictional claims in published maps and institutional affiliations.

Copyright: (C) 2022 by the authors. Licensee MDPI, Basel, Switzerland. This article is an open access article distributed under the terms and conditions of the Creative Commons Attribution (CC BY) license (https:// creativecommons.org/licenses/by/ $4.0 /)$.
1 Department of Biotechnology, KLE Technological University, Hubbali 580031, India

2 Department of Pharmaceutics, College of Pharmacy, King Saud University, Riyadh 11451, Saudi Arabia; salshehri1@ksu.edu.sa

3 Department of Pharmacy Practice, College of Pharmacy, AlMaarefa University, Dariyah 13713, Saudi Arabia; mghoneim@mcst.edu.sa (M.M.G.); bmannasaheb@mcst.edu.sa (B.A.M.)

4 Department of Pharmaceutical Chemistry, College of Pharmacy, Najran University, Najran 66462, Saudi Arabia; matermaha@gmail.com

5 Department of Clinical Laboratory Sciences, College of Applied Medical Sciences, Najran University, Najran 66462, Saudi Arabia; maalshahrani@nu.edu.sa

6 Department of General Science, Ibn Sina National College for Medical Studies, Al Mahajar Street, P.O. Box 31906, Jeddah 21418, Saudi Arabia; aeju_kh@yahoo.com

7 Department of Pharmaceutical Chemistry, Ibn Sina National College for Medical Studies, Al Mahajar Street, P.O. Box 31906, Jeddah 21418, Saudi Arabia; alibahfi@hotmail.com

8 School of Basic and Applied Sciences, Dayananda Sagar University, Bangalore 560078, Karnataka, India; sunilsmorey@gmail.com

9 Department of Pharmacology, College of Pharmacy, Najran University, Najran 66462, Saudi Arabia; i.ibrahimshaikh09@gmail.com

10 Clinical and Hospital Pharmacy Department, College of Pharmacy, Taibah University, Al-Madinah Al-Munawwarah 41311, Saudi Arabia; nbinothman@taibahu.edu.sa

11 Department of Clinical Pharmacy, School of Pharmacy, Management and Science University, University Drive, Off Persiaran Olahraga, Shah Alam 40100, Selangor, Malaysia

12 Department of Microbiology and Immunology, Ibn Sina National College for Medical Studies, Jeddah 21418, Saudi Arabia; muashe@yahoo.co.in

13 Department of Pharmaceutics, College of Pharmacy, Najran University, Najran 66462, Saudi Arabia; zaki.manipal@gmail.com

* Correspondence: uday1232002@yahoo.com (U.M.M.); shakeeliqubal@gmail.com (S.M.S.I.)

Abstract: Bionanotechnology is a branch of science that has revolutionized modern science and technology. Nanomaterials, especially noble metals, have attracted researchers due to their size and application in different branches of sciences that benefit humanity. Metal nanoparticles can be synthesized using green methods, which are good for the environment, economically viable, and facilitate synthesis. Due to their size and form, gold nanoparticles have become significant. Plant materials are of particular interest in the synthesis and manufacture of theranostic gold nanoparticles (NPs), which have been generated using various materials. On the other hand, chemically produced nanoparticles have several drawbacks in terms of cost, toxicity, and effectiveness. A plant-mediated integration of metallic nanoparticles has been developed in the field of nanotechnology to overcome the drawbacks of traditional synthesis, such as physical and synthetic strategies. Nanomaterials' tunable features make them sophisticated tools in the biomedical platform, especially for developing new diagnostics and therapeutics for malignancy, neurodegenerative, and other chronic disorders. Therefore, this review outlines the theranostic approach, the different plant materials utilized in theranostic applications, and future directions based on current breakthroughs in these fields.

Keywords: gold; cytotoxicity; antioxidant; anticancer; antibacterial; antifungal 


\section{Introduction}

Gold nanoparticles (AuNPs), due to their unique qualities and various surface characteristics, have been widely exploited in bionanotechnology. The ease with which AuNPs can be functionalized makes them a flexible platform for nano biological assemblies containing oligonucleotides [1], antibodies [2], and proteins [3]. AuNPs bioconjugates have also emerged as attractive options for developing novel biomaterials for biomedical research. The versatility of AuNPs has made them useful in various biomedical applications. The binding of the sample to the AuNPs can change the rheological feature of AuNPs, such as surface plasmon resonance, conductivity, and redox behavior, resulting in notable signals [4-7] in diagnostics. With their enormous surface area, AuNPs can also be used as a platform for therapeutic agents. Nanotechnology has been in existence for thousands of years. Ancient people used to stain their drinking glasses with nanoparticles [8]. The divergence of nanotechnology within other fields of science and further innovations have made a significant impact on biotechnology, medicine, pharmaceutics, physics, chemistry, and optics, etc. There is evidence that metals are present in living systems in different forms, playing a significant role in various biochemical processes, growth metabolism, and healing [9]. Blood contains the Hema protein, $\mathrm{Zn}, \mathrm{Mn}, \mathrm{Cu}$, and other vital trace metals in the biological system.

This review aims to summarize the data on gold nanoparticles synthesized by extracts of medicinal plants, their parts, and their usefulness in biological and theranostic properties.

\subsection{Nanoparticle Synthesis}

Nanoparticle synthesis from metals has gained enormous interest among researchers because of nanoparticles' diverse application in many fields such as cancer therapy, drug delivery, food safety, fabrics, chemistry, water treatment, and photocatalysis, as well as because of their antioxidant, antibacterial, and cytotoxic properties [10-20]. The uses of nanoparticles in various fields are possible because of several factors, including the nanoparticles' shape, size, distribution, and surface plasmon [21-23].

Nanoparticles have been used for thousands of years without knowledge concerning the exact phenomenon and synthesis [8]. Drinking glasses in ancient times were coated with $\mathrm{Au}$ nanoparticles and were synthesized following three primary methods: physical, chemical, and biological methods.

\subsection{Physical Method}

The advantages of the physical synthesis method are the absence of a solvent, which is hazardous to the environment, and the uniformity of the nanoparticles produced by the physical methods. The tube furnace method of synthesis occupies an ample space, and an enormous quantity of heat is required to raise the temperature of the furnace. Several minutes are necessary to preheat the furnace [24]. A small ceramic heater with a local heating chamber could be used to synthesize Ag nanoparticles [25]. As a result, the formed nanoparticles were reported to have a mean geometric diameter that was spherical without agglomeration [26]. The advantage of the laser ablation method, in comparison to other techniques, is that it is free from chemical reagents. The purity of the nanoparticles was assured in this method [27]. The nanoparticles produced by the discharge method used to fabricate Ag nanoparticles $[28,29]$ had $99.99 \%$ purity. The purity and size distribution were uniform when compared to other forms of synthesis.

\subsection{Chemical Synthesis of Gold Nanoparticles}

In recent years, a solution-based strategy for controlling the size, shape, and surface functionality has been created [30-32]. In 1951, a new method for synthesizing AuNPs was devised by boiling hydrogen tetrachloroaurate $\left(\mathrm{HAuCl}_{4}\right)$ with citric acid [33]. Citrate has a lowering and stabilizing effect [34]. To adjust particle size, Frens developed the process by modifying the gold-to-citrate ratio [33]. This approach has been commonly used to make dilute solutions of relatively stable spherical AuNPs with diameters of 10 to $20 \mathrm{~nm}$; however, 
bigger AuNPs (e.g., $100 \mathrm{~nm}$ ) have also been made. These citrate-stabilized AuNPs may undergo irreversible aggregation during the functionalization process with thiolate ligands. Several solutions have been devised to tackle this difficulty, including using a biosurfactant, Tween 20. Similarly, a two-step method for functionalizing gold nanoparticles was made by reducing tetrachloroauric acid in water with trisodium citrate. The physisorbed chloride and citrate on gold nanoparticles are first displaced by thioctic acid, which is then replaced by thiols with the desired functionality in the second step [35,36]. The demand for high dilution, on the other hand, makes large-scale manufacture difficult.

AuNPs synthesis [37] was conducted in 1994 to produce organic soluble alkanethiolstabilized AuNPs by adopting a biphasic reduction, with the use of tetraoctylammonium bromide as a phase transfer reagent and sodium borohydride $\left(\mathrm{NaBH}_{4}\right)$ as a reducing agent [37]. By changing response variables such as the gold-to-thiol ratio, the reduction rate, and the reaction temperature, this technique yields low-dispersity AuNPs ranging from 1.5 to $5 \mathrm{~nm}$ [38]. The synergic impact of thiol-gold generated strong connections and Van der Waals attractions between the adjacent ligands, giving these alkanethiol-protected AuNPs better stability than most other AuNPs [39].

\section{Biological Method of Synthesis}

Although the chemical synthesis of metallic nanoparticles is a standard procedure, the cost and hazardous effects of reducing reagents and stabilizing agents restrict their use. Furthermore, in biomedical applications, these nanoparticles could be toxic [40,41]. As a result, ecologically friendly and cost-effective nanoparticle synthesis techniques that do not rely on harmful chemicals are needed. In recent years, biological nanoparticle production has gained popularity as a green and environmentally friendly process [42]. Plants or plant extracts and microorganisms and enzymes were employed to synthesize nanoparticles using a natural method $[43,44]$. The proposed synthetic mechanism for plantmediated synthesis of gold nanoparticles is depicted in Figure 1A.

Plants are increasingly being used to synthesize nanoparticles because of their widespread availability, low cost, environmental friendliness, and non-toxic nature. Plants such as Azadirachta indica have recently been used to study the production of AuNPs. Medicago sativa, Aloe vera, Cinnamomum camphora, Pelargonium graveolens, Coriandrum sativum, Coriandrum sativum, Lemongrass, Terminalia catappa, and Terminalia catappa have all been reported [41,45-51].

Many scientists are experimenting with the production of AuNPs from plant extracts as biomedicines against drug-resistant bacteria. Arunachalam et al., 2013, proposed using Memecylon umbellatum nanoparticles as chemical sensors [52]. Kalishwaralal et al., 2010, showed how a bacterium, Brevibacterium casei, can synthesize and stabilize spherical-shaped $\mathrm{Au}$ and Ag nanoparticles in an unprecedented green process. The biological activities of the produced particles were confirmed based on their durable anti-coagulant actions. Similarly, Citrus limon, Citrus reticulata, and Citrus sinensis, all citrus fruits, as well as Piper pedicellatum, have been synthesized as polymorphic gold nanoparticles with promising biological uses. These chemical constituents can operate as a reducing, stabilizing, and capping agent [52-57]. Chebula Terminalia, Memecylon edule, and Nyctanthes arbor-tristis flower extract have potential medicinal and industrial applications. Murraya koenigii and Musa paradisiaca show antibacterial activity; Mangifera indica, Cochlospermum gossypium, and Cinnamomum zeylanicum photoluminescent particles are used for the production of noble metal nanoparticles, which enable much faster synthesis and colloidal stability comparable to those of chemical reduction [58-67]. 


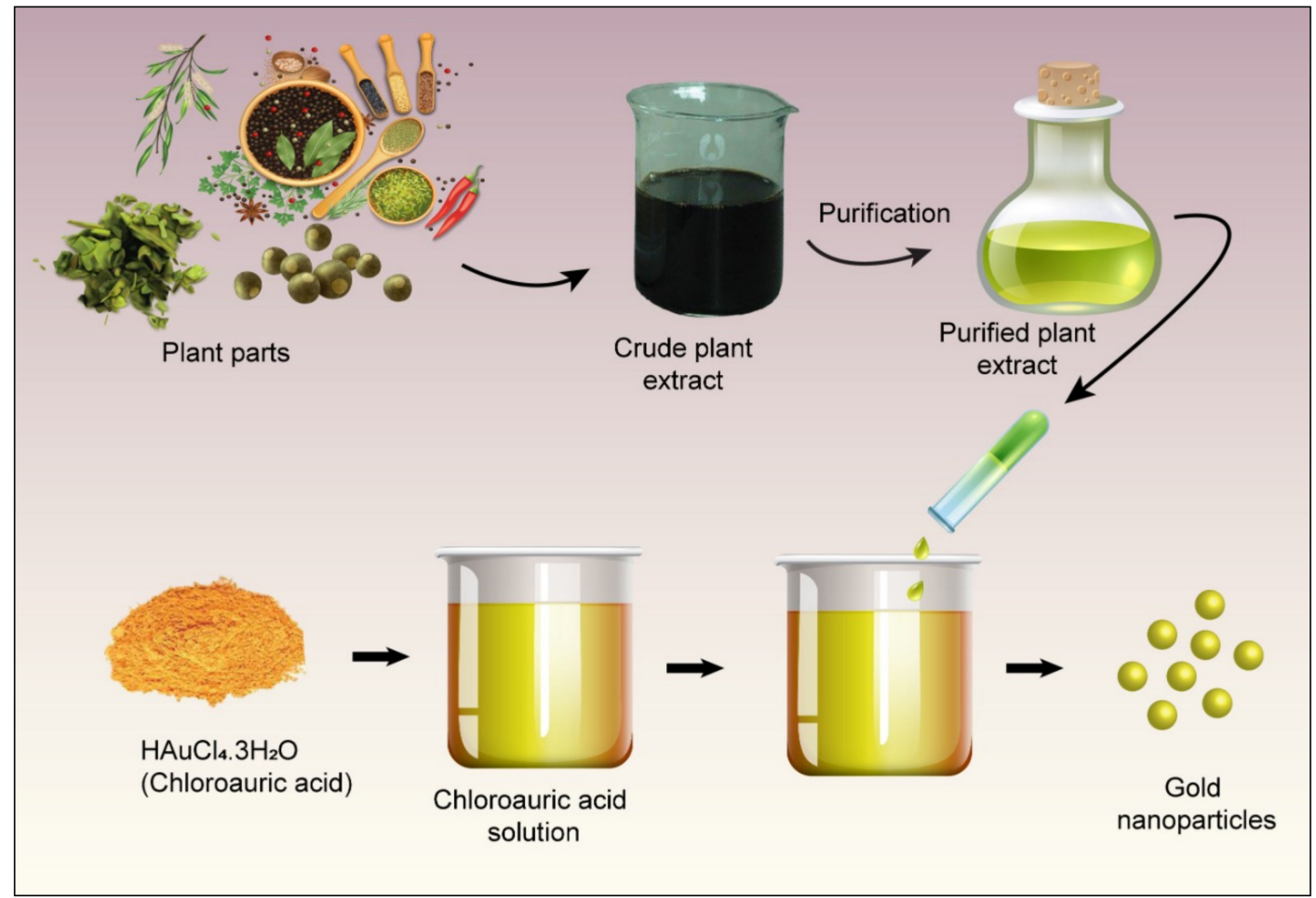

(A)

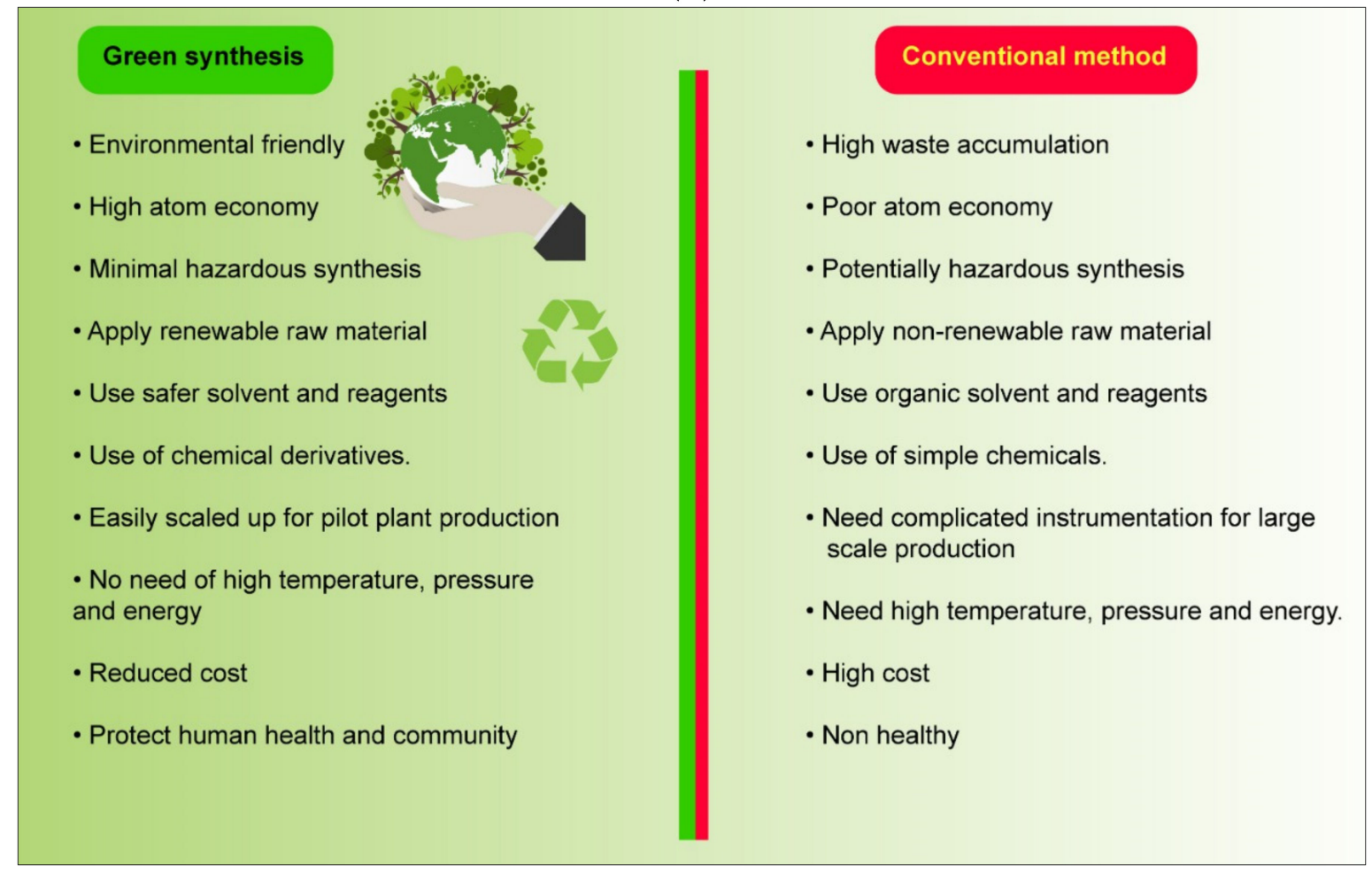

(B)

Figure 1. (A) Proposed synthetic mechanism for plant-mediated synthesis of gold nanoparticles. (B) The advantages of green synthesis over conventional methods.

\subsection{Green Synthesis of Gold Nanoparticles}

Many metal nanoparticles synthesized via the green process possess several advantages, as shown in Figure 1B. Their unique physicochemical properties, high surface-tovolume ratio, low cost of synthesis, and surface functionalization were reported by Ankit 
Kumar Singh. Additionally, this review found that several studies have reported in detail a variety of plants and plant parts used in metal nanoparticle generation: the bark of Mimusops elengi was used to synthesize Au nanoparticles; bimetallic nanoparticles were synthesized using Azadictira Indica leaf extract; Au nanoparticles were synthesized from natural rubber; and Aelovera plant extract and lemongrass extract have applications on infrared-absorbing coating [68-73]. The antioxidant, anti-inflammatory, antidiabetic, and antibacterial activities of Holopetelea integrifolia leaf extract were studied, and synthesized Au nanoparticles from Halymenia dilatata were studied regarding their antioxidant, anticancer, and antibacterial activities; synthesized conjugated Au nanoparticles from Nerium oleander were studied regarding their anticancer activity against MCR-7 cell lines [74-76]. The anticancer activity of Au nanoparticles synthesized using Lonicera japonica was also studied. Ag and Au nanoparticles synthesized from Pleuropterus multiflorus roots were investigated regarding their anticancer activity against the A549 lung cancer cell line [77,78]. Au nanoparticles synthesized using the Mucuna pruriens plant extract were studied regarding an antiparkinsonian drug, and it was reported that poly-shaped Au nanoparticles were synthesized using Saraca indica bark extract and were studied regarding catalytic reduction. The anticancer activity of Ag and Au nanoparticles synthesized using Dendropanax morbifera leaf extract was studied, as well as the antimicrobial characteristics of $\mathrm{Au}$ and Ag nanoparticles using Trianthema decandra extract. The antioxidant and anticancer properties of Au nanoparticles synthesized using Antigonon leptopus leaf extract were studied, and the anticancer activities of noble metal nanoparticles using Psidium guajava leaf extract and Syzygium aromaticum bud extract were studied. The antibacterial properties of Au nanoparticles synthesized from Nepenthes khasiana leaf extract were investigated, as well as Au nanoparticles synthesized from Schisandra Chinensis fruit extract. Ag and Au nanoparticles synthesized using Dalbergia sissoo leaf extract were studied, and Ag nanoparticles synthesized from Cassia italica leaf extract were also studied. The kinetics of the Au nanoparticles synthesized using Camellia chinesis leaves and leaf buds were studied, as well as the apoptotic effects of Au nanoparticles synthesized using Curcuma wenyujin [79-87].

\subsection{Medicinal Plants}

Nature's contribution to the health of human beings is unimaginable. A wide variety of plants are used in curing diseases and for a healthy lifestyle. India has a rich source of medicinal plants used for various purposes. More than 17,000 species are used as medicinal plants in India. The constituents/drugs present in medicinal plants are called phytochemicals. These phytochemicals act on the biochemical processes in animals, human beings, and microbes. The properties of phytochemicals are used due to their antioxidant, antimicrobial, and anti-inflammatory properties [88-90].

The World Health Organization (WHO) indicated that traditional remedies are used by $80 \%$ of the world's population. For a long time, plants have been used as medicine in India's alternative medical systems, such as Unani, Ayurveda, Siddha, Yoga, and homeopathy. Plant-derived medicines are alternatives to synthetic drugs, gaining importance in modern medicine. In the developing world, primary health care services have benefited from medicinal plants. In the Ayurvedic medical system, many plants and plant-based materials are employed to treat ailments. A treatise on Ayurvedic medicine called "Charaka Samhitha" mentions over 700 herbs [91-97]. Several therapeutic plants are mentioned in the Vedas, such as the Rig Veda and the Atharva Veda.

AuNPs are well-known nanomaterials with a wide range of biomedical applications. AuNPs can be synthesized using a variety of microbes and plants, mainly through the use of fruit extracts. Fruit extracts are used because they naturally concentrate chemicals with medicinal effects. Studies have shown that UV-visible spectroscopy, transmission or scanning electron microscopy, dynamic light scattering, and Fourier transformation infrared spectroscopy techniques are the methods most often used to characterize AuNPs and capping biomaterial. Figure 2 shows some of the important outcomes in gold nanoparticles obtained from plant components. 


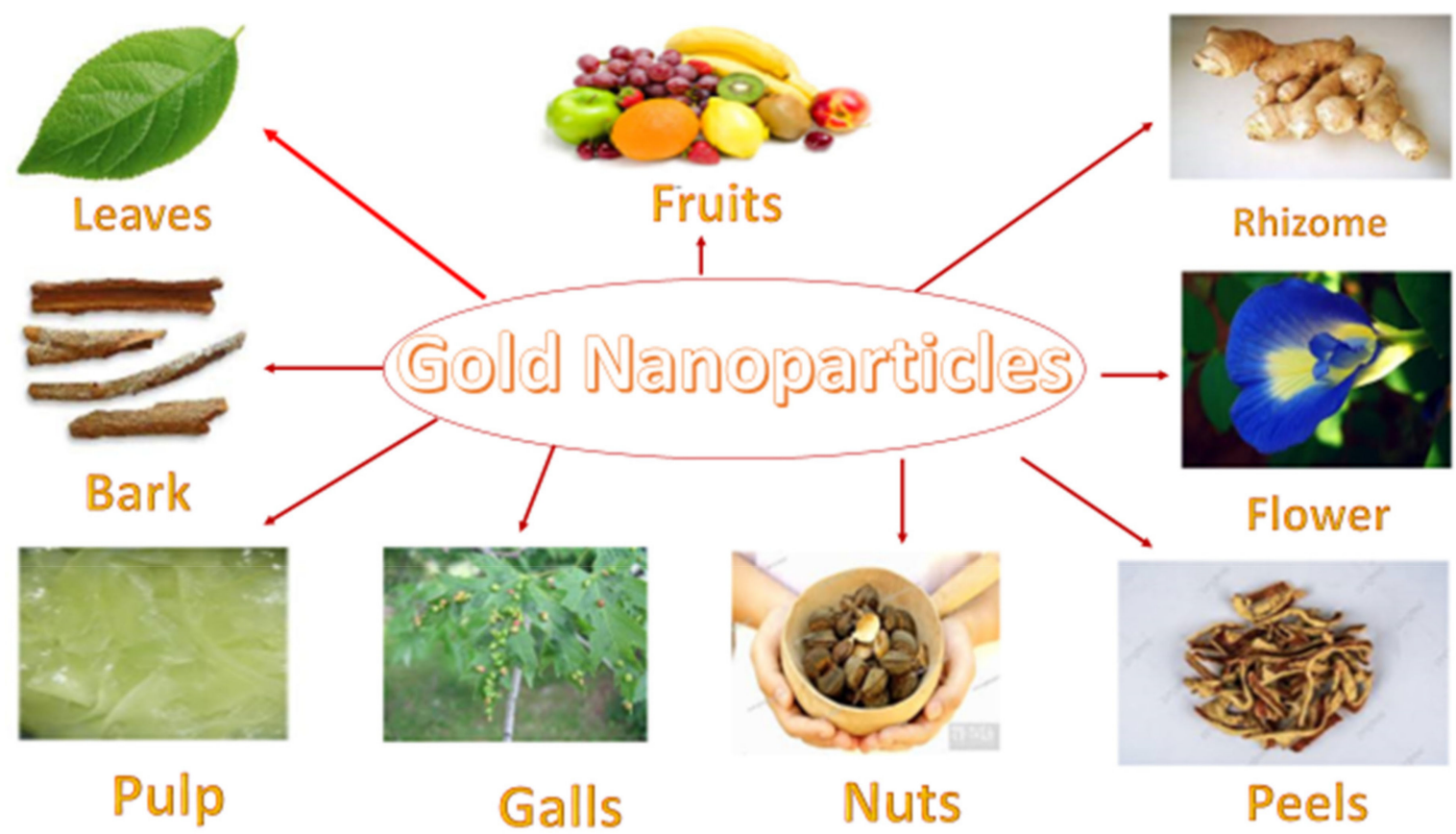

Figure 2. Plant parts extract can be used for the biosynthesis of gold nanoparticles.

\section{Characterization}

\subsection{UV-Visible Spectroscopic Analysis}

In an aqueous solution, gold nanoparticles synthesized from various plant parts were measured using a UV photometer and a Lab India UV3000 spectrophotometer, which read at $450 \mathrm{~nm}$ and $650 \mathrm{~nm}$ for the Au nanoparticles. Readings were taken every 30 min for $6 \mathrm{~h}$. The absorbance and transmittance of the Au nanoparticles were measured at $450 \mathrm{~nm}$ to $650 \mathrm{~nm}$ using $3 \mathrm{~mL}$ of each sample in a cuvette, and they were subjected to spectral analysis. At 520-560 nm, a single, narrow absorbance band was found, which is typical of the production of tiny gold nanoparticles [98-103], and this was validated by the TEM results as shown in Figure 3.

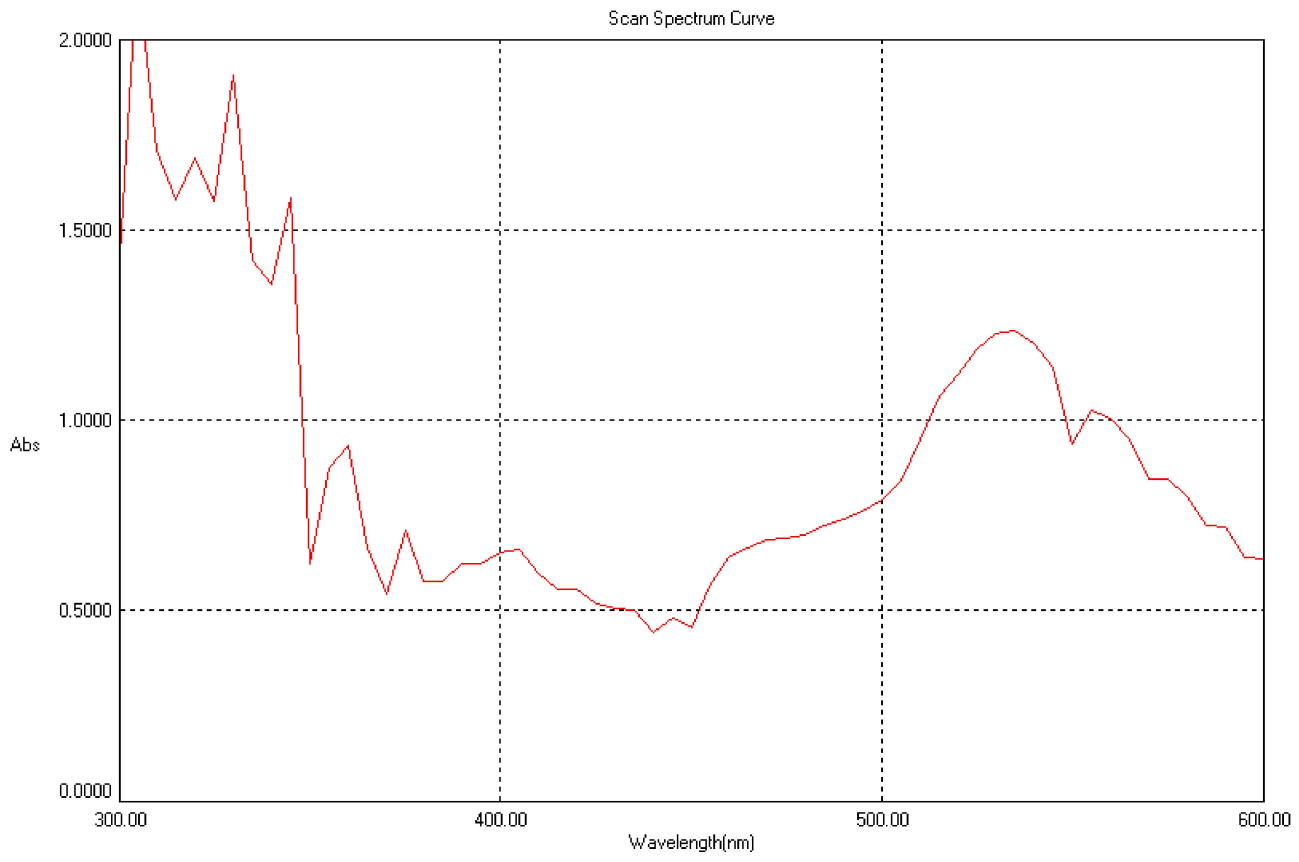

Figure 3. UV-spectral analysis for gold nanoparticles. 


\subsection{Fourier Transform Infrared Spectroscopic Analysis (FTIR)}

A total of $5 \mathrm{gm}$ of each synthesized freeze-dried Au nanoparticle from different plants parts was taken and pressed with $0.2 \mathrm{gm}$ of $\mathrm{KBr}$ pellets to measure the infrared radiation spectrum (IR) examined under an FTIR spectrophotometer (JASCO) over wavelengths in the range of $4000 \mathrm{~cm}^{-1}-400 \mathrm{~cm}^{-1}$. The FTIR spectrum of the green synthesized AuNPs is shown in Figure 4. The strong bands at $3389 \mathrm{~cm}^{-1}$ (O-H stretching alcohol), $2919 \mathrm{~cm}^{-1}$ (C-H stretching alkane) and $2844 \mathrm{~cm}^{-1}$ (C-H stretching aldehyde) were due to the reduction of $\mathrm{Au}^{3+}$ to $\mathrm{Au} 0$. A band at $1458 \mathrm{~cm}^{-1}$ corresponds to an $\mathrm{NH}$ bend, and the very broad band of $\mathrm{NH}^{+3}$ stretch was observed in the $3000-3500 \mathrm{~cm}^{-1}$ range. The peaks at $1700 \mathrm{~cm}^{-1}$ (C-C stretching alkane), $1374 \mathrm{~cm}^{-1}$ (O-H bending phenol), and $1162 \mathrm{~cm}^{-1}$ (CO-O- CO stretching anhydride) confirm the capping biomaterials of phytochemicals from plant extracts such as polyphenols, flavonoids, and terpenoid compounds. Similar reports of FTIR peaks for phenols and flavonoids from gold nanoparticles biosynthesized from Cissus quadrangular extract confirm the capping biomaterial of the synthesized nanoparticles. The bands at $1261 \mathrm{~cm}^{-1}$ and $1034 \mathrm{~cm}^{-1}$ are typically assigned to the vibration of ribose (C-C sugar), which correspond to an epoxy bond, semi-acetal, and primary alcohol, respectively. Further, the bands at 2920 and $1374 \mathrm{~cm}^{-1}$ correspond to methylene stretching and methyl deformation vibrations, respectively.

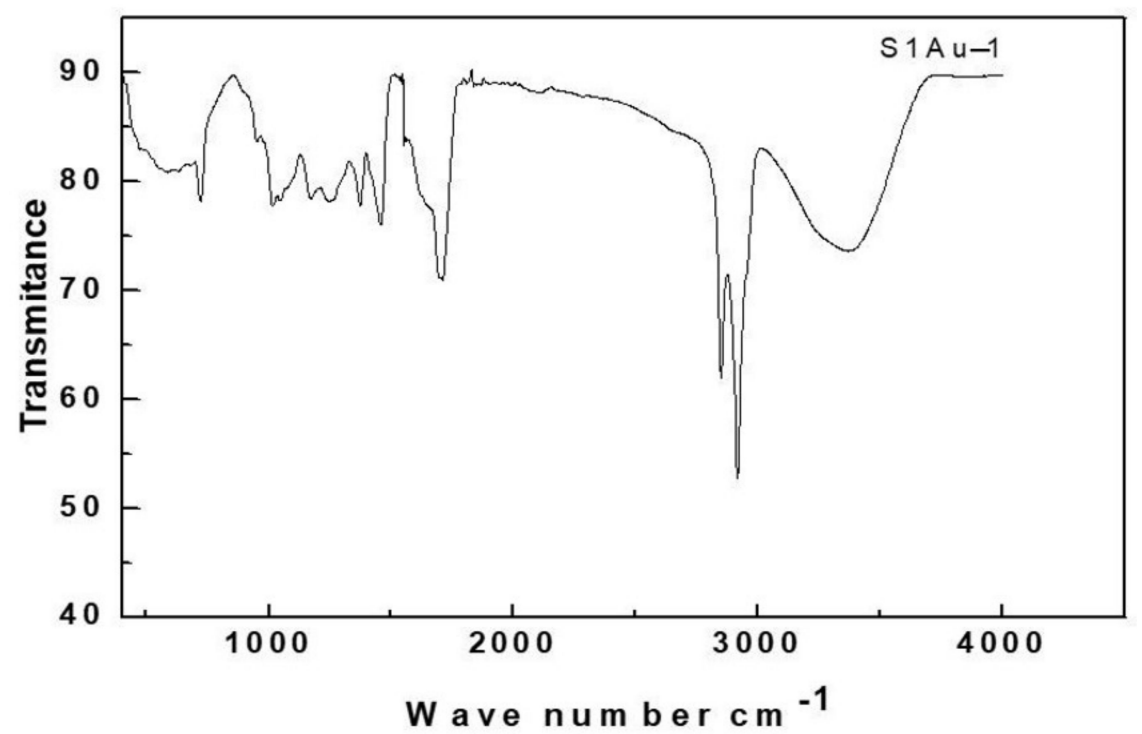

Figure 4. FTIR for gold nanoparticles.

For example, the gold nanoparticles produced using plant extracts had bands at $617 \mathrm{~cm}^{-1}$, $1125 \mathrm{~cm}^{-1}, 1376 \mathrm{~cm}^{-1}, 1658 \mathrm{~cm}^{-1}$, and $3278 \mathrm{~cm}^{-1}$ in their FTIR pattern [104-109]. The aromatic hydroxyl and benzene rings were assigned bands at $3402 \mathrm{~cm}^{-1}, 1606 \mathrm{~cm}^{-1}$, and $1518 \mathrm{~cm}^{-1}$, indicating that the extract contains phenols. The bands at $2931 \mathrm{~cm}^{-1}$ and $1402 \mathrm{~cm}^{-1}$ correspond to methylene stretching and methyl deformation vibrations, respectively, whereas the sugar content is shown by bands at $1260 \mathrm{~cm}^{-1}, 1113 \mathrm{~cm}^{-1}$, and $1076 \mathrm{~cm}^{-1}$, which correspond to an epoxy bond, semi-acetal, and primary alcohol, respectively.

\subsection{Transmission Electron Microscope (TEM)}

The synthesized Au nanoparticles were loaded separately into the FEI. A Tecnai G2 F20 STFE-TEM microscope was used. The sample was dried by pressing with blotting paper to remove excess water and loaded onto the carbon-coated copper grid. The TEM was operated at $200 \mathrm{Kv}$, with a resolution of $0.24 \mathrm{~nm}$, and Cs of $1.2 \mathrm{~nm}$; the shape and size were determined as shown in Figure 5. The high-resolution TEM images show agglomerated polycrystalline particles, and the SAED confirmed the face-centered cubic (FCC) structure 
incorporation of the poly-dispersed XRD pattern. The EDX analysis proved the presence of only Au metal, and no other elements were present.

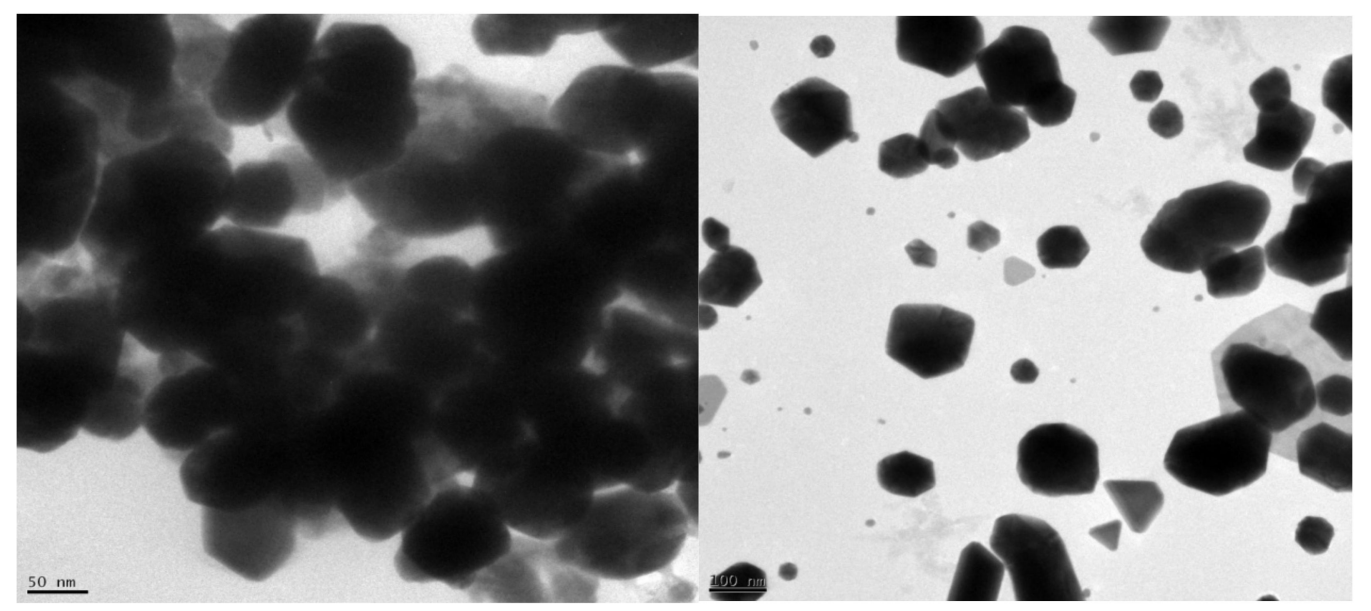

Figure 5. TEM analysis for gold nanoparticles.

SEM, TEM, and AFM are the most commonly utilized microscopic techniques for morphological analyses of nanoparticles. The application of these microscopic methods in nanoparticle morphology research has already been mentioned. TEM has a higher magnification and resolution than the SEM. The electron diffraction pattern for a specified region (SAED) is also utilized in TEM to distinguish crystalline structures from amorphous structures [105,110]. The shape of the gold nanoparticles is studied using AFM [109-111].

\subsection{X-ray Diffraction (XRD)}

The Analytical Expert MRD, the model instrument, is generally utilized to investigate the characteristics of synthetic Au nanoparticles for samples. The fine powder of nanoparticles is loaded onto the XRD sample holder separately, and readings are recorded. The size of the $\mathrm{Au}$ nanoparticles is calculated using Debye-Scherer's equation: $\mathrm{D}=0.9 \lambda / \beta \cos \theta$, where $\mathrm{D}$ is the average crystallite size. $\mathrm{X}$ is the XRD wavelength $(1.54 \mathrm{~nm})$; $\mathrm{B}$ is the (FWHM), and $\theta$ is the Bragg angle. The plant-mediated synthesized XRD characterized the Au nanoparticles. The diffraction peak $2 \theta$ values assigned at $38.2^{\circ}, 44.4^{\circ}$, and $64.6^{\circ}$ were denoted as the (111), (200), and (220) planes, respectively. The planes agree well with the JCPDS card: 04-0784 data. The XRD pattern determined the intensity of the peak, the peak position, the width, and the full width at half-maximum (FWHM) as shown in Figure 6. The XRD data revealed that the nanoparticles are crystalline and face-cantered cubic (fcc). The particle mean size was determined using Debye-Scherer's formula to determine the average size of the Au particles. The high-energy X-rays can penetrate the materials deeply and reveal important details about the bulk structure. The Debye-Scherrer equation computes the crystallite sizes using the XRD technique. The usage of XRD patterns/peaks during gold nanoparticle production has been reported [98,104,110-114]. 


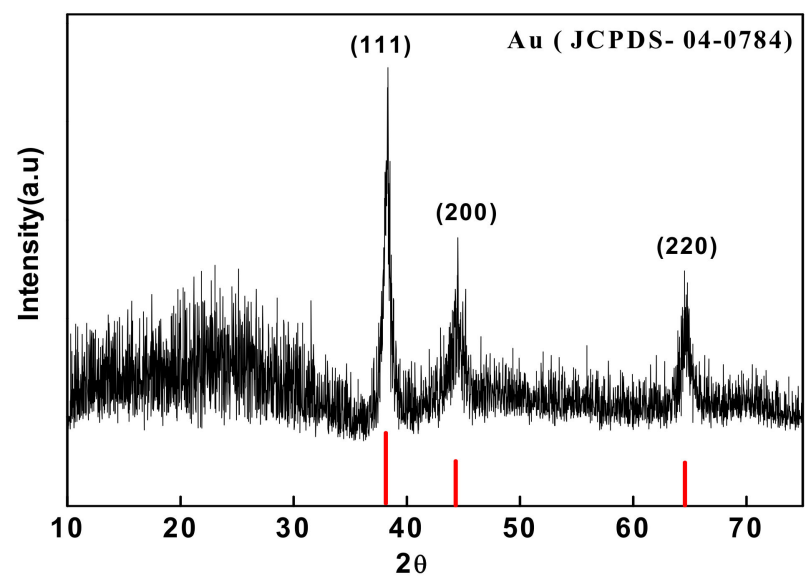

Figure 6. XRD analysis for gold nanoparticles.

\section{Theranostic Applications}

Nanoparticles are associated with a small stature and shape. The surface-to-volume ratio of nanoparticles is very high, which leads to enhanced electrical, optical, magnetic, antifungal, antioxidant, antibacterial, anti-inflammatory, and anticancer properties $[115,116]$. The surfaceto-volume ratio offers many perspectives for the food sector. Nanoparticles' are critical and significant in applications in biomedicine, especially in treating cancer, the diagnosis of HIV, and the proliferation of cancer cells. In 1918, scientists made drastic progress in finding the function of various metal nanoparticles in biological systems [117-119]. Metal nanoparticles, mainly gold, are used in medicine for diagnosis, targeting, and therapeutics (Figure 7).

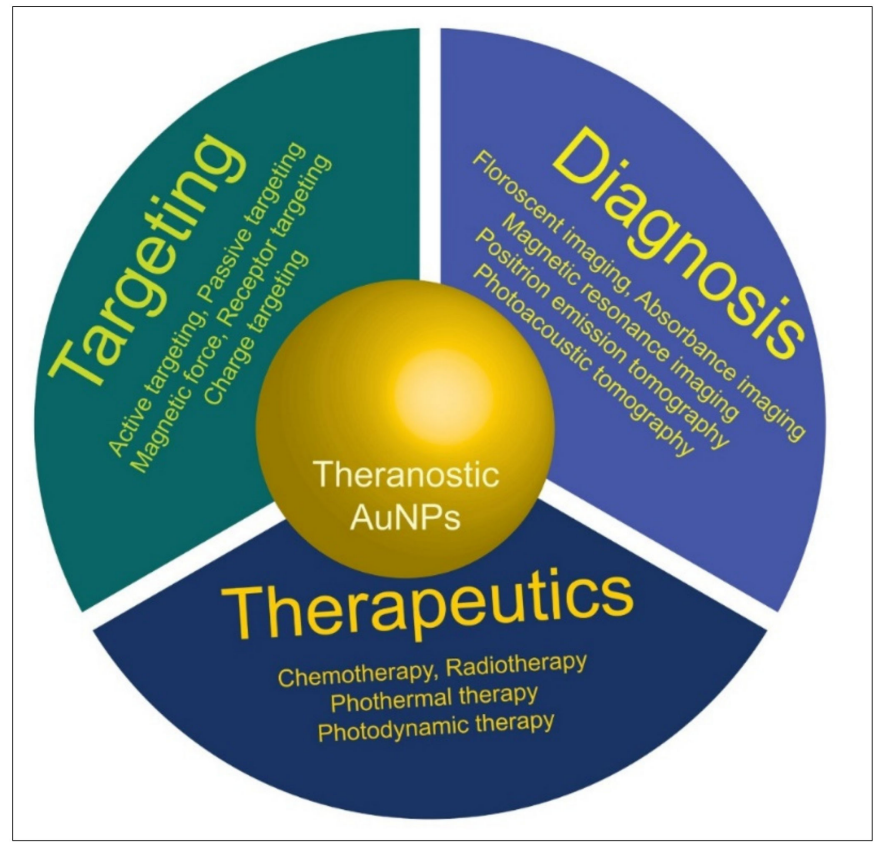

Figure 7. Theranostic applications of AuNPs in medical sciences and applied fields.

\subsection{Principle of MTT Assay}

Tetrazolium salt reduction is now universally acknowledged as a reliable method of examining cell growth. MTT (3-(4, 5-dimethylthiazolyl-2)-2, 5-diphenyltetrazolium bromide) is a yellow tetrazolium reduced by metabolically active cells, in part via dehydrogenase enzymes, to generate reducing equivalents such as NADH and NADPH. The intracellular purple formazan that results can be solubilized and measured using spectrophotometric 
methods (Figure 8). The assay evaluates the cell proliferation rate and, conversely, cell viability reduction induced by metabolic processes such as apoptosis or necrosis [120].

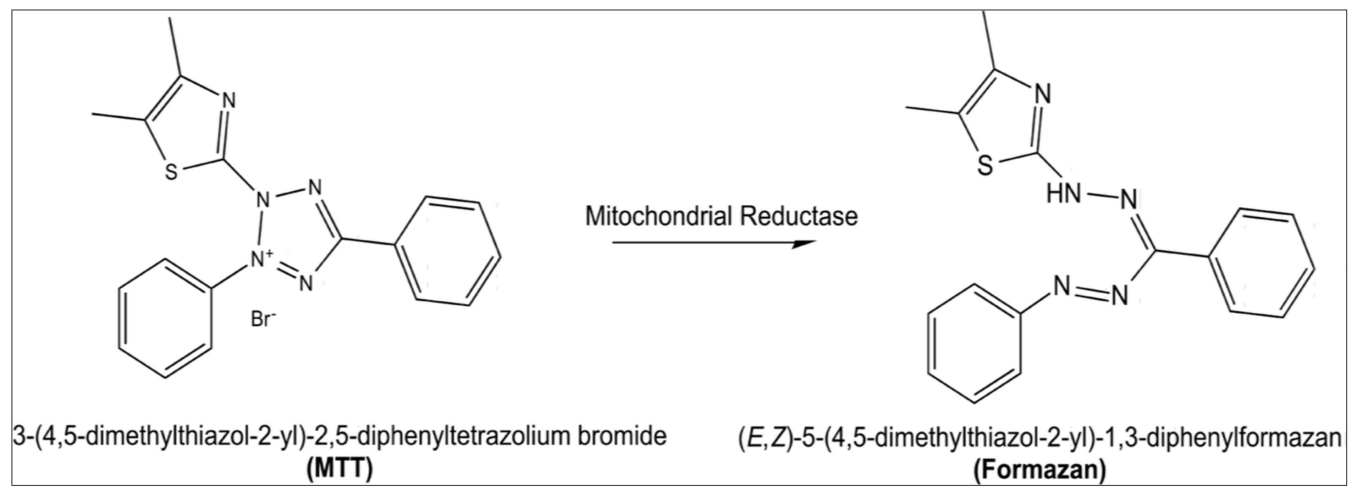

Figure 8. Principle of MTT assay.

\subsection{Biological and Theranostic Applications}

As shown in Table 1, many researchers have demonstrated that AuNPs can successfully attack cancer cells. AuNPs derived from Gymnema Sylvestre, often known as cowplant, were cytotoxic to Hep2 cells. After treatment with AuNPs, Hep2 cells showed morphological alterations. Increases in reactive oxygen species levels and alterations in the nucleus were discovered, implying that apoptosis was responsible for the demise of Hep2 cells [121]. Another cervical cancer cell type, the HeLa cell line, also reacted to AuNPs. Rounding, shrinkage, and granulation were identified as morphological alterations. The AuNPs' activity was attributable to the NPs' ability to penetrate the cell membrane efficiently. AuNPs have caused responses from other tumor cells, including Ehrlich's ascites carcinoma, breast cancer cells, and MCF-7 cells. Green tea polyphenols were used in the production of AuNPs. AuNPs synthesized from green tea and AuNPs synthesized from epigallocatechin-3-gallate were compared. Both AuNPs were able to trigger apoptosis in tumor cells while preventing tumor cell damage in normal hepatocytes. Green-generated AuNPs, on the other hand, demonstrated improved tumoricidal and hepatoprotective effects. When AuNPs generated by Actinidia deliciosa were examined on HCT-116 cells using an MTT assay, they showed 71 percent activity at their highest concentration $(350 \mathrm{~g} / \mathrm{mL})$. The cytotoxic effect of the AuNPs was shown to be concentration dependent [122].

Table 1. Biological and theranostic applications of gold nanoparticles.

\begin{tabular}{|c|c|c|c|c|c|c|}
\hline S. No & Name of the Plant & Activity & Cell Line Used & Shape & Size $(\mathrm{nm})$ & Ref. \\
\hline 1 & \multicolumn{5}{|c|}{ Nanoparticles with antibacterial activity } & \\
\hline 1.1 & Areca catechu & Antibacterial & - & Spherical & 13 & [123] \\
\hline 1.2 & Acorus calamus & Antibacterial & - & Spherical & 100 & [124] \\
\hline 1.3 & Ananas comosus & Antibacterial & - & Spherical & 16 & [125] \\
\hline 1.4 & Benincasa hispida & Antibacterial & - & Spherical & 23 & [126] \\
\hline 1.5 & Brazilian red propolis & Antibacterial & - & $\begin{array}{c}\text { Rods, triangular, } \\
\text { pentagonal, hexagonal }\end{array}$ & $8-15$ & [127] \\
\hline 1.6 & $\begin{array}{c}\text { Clitoria ternatea (Asian } \\
\text { pigeonwings) }\end{array}$ & Antibacterial & - & $\begin{array}{l}\text { Spherical, triangular, } \\
\text { hexagonal }\end{array}$ & 10 & [128] \\
\hline 1.7 & Citrus maxima & Antibacterial & - & Spherical & $27-30$ & [104] \\
\hline 1.8 & Coreopsis lanceolate & $\begin{array}{l}\text { Detections of } \\
\text { aflatoxins }\end{array}$ & - & Sphere & $23-30$ & [129] \\
\hline 1.9 & Caesalpinia pulcherrima & Antibacterial & - & Spherical & $10-50$ & [130] \\
\hline
\end{tabular}


Table 1. Cont.

\begin{tabular}{|c|c|c|c|c|c|c|}
\hline S. No & Name of the Plant & Activity & Cell Line Used & Shape & Size $(\mathrm{nm})$ & Ref. \\
\hline 1.10 & Carthamus tinctorius $L$ & Antibacterial & - & Triangular, spherical & $40-200$ & [131] \\
\hline 1.11 & Catharanthus roseus & Antibacterial & - & Spherical, triangular & $3-9$ & [132] \\
\hline 1.12 & Carica papaya & Antibacterial & - & Spherical, triangular & $2-20$ & [133] \\
\hline 1.13 & Coleus forskohlii & Bactericidal activity & - & Triangular & $25-40$ & [134] \\
\hline 1.14 & Ceiba pentandra $(L)$ & Antibacterial & - & Spherical & $20-48$ & [135] \\
\hline 1.15 & Diospyros ferrea & Antibacterial & - & Diverse & $70-90$ & [136] \\
\hline 1.16 & Dioscorea batatas & Antibacterial & - & Diverse & $19-56$ & [137] \\
\hline 1.17 & Dimocarpus longan & Antibacterial & - & Diverse & 25 & [138] \\
\hline 1.18 & Dracocephalum kotschyi & Antibacterial & - & Spherical & 11 & [139] \\
\hline 1.19 & Euphorbia hirta & Antibacterial & - & Spherical & $6-7$ & [140] \\
\hline 1.20 & Gloriosa superba & Antibacterial & - & Spherical & 25 & [141] \\
\hline 1.21 & Galaxaura elongate & Antibacterial & - & $\begin{array}{l}\text { Rod, triangular, } \\
\text { hexagonal }\end{array}$ & $3-77$ & [142] \\
\hline 1.22 & Bay cedar & Antibacterial & - & Spherical & $20-25$ & [143] \\
\hline 1.23 & Hibiscus cannabinus & Antibacterial & - & Spherical & 13 & [144] \\
\hline 1.24 & Hoveniadulcis & Antibacterial & - & Spherical & 20 & [145] \\
\hline 1.25 & Helianthus annuus & Antibacterial & - & Polydispersed & 35 & [146] \\
\hline 1.26 & Hevea brasiliensis & $\begin{array}{c}\text { Cytotoxicity } \\
\text { and genotoxicity }\end{array}$ & CHO-K1 cells & Spherical, triangular & 50 & [147] \\
\hline 1.27 & Justica wynaadensis & Antibacterial & - & Spherical & $30-50$ & [148] \\
\hline 1.28 & Jasminum auriculatum & Antibacterial & - & Spherical & $8-37$ & [149] \\
\hline 1.29 & Lobila nicotianifolia & Antibacterial & - & Spherical & 80 & [150] \\
\hline 1.30 & Mammea suriga & Antibacterial & - & Square & 50 & [151] \\
\hline 1.31 & Mentha piperita & Antibacterial & - & Hexagonal & 78 & [152] \\
\hline 1.32 & Maytenus royleanus & $\begin{array}{l}\text { Antibacterial, } \\
\text { Leshmenia }\end{array}$ & - & Hexagonal & 30 & [153] \\
\hline 1.33 & Musa paradisiaca (Banana) & Antibacterial & - & Diverse & 300 & [154] \\
\hline 1.34 & Nepenthes khasiana & Antibacterial & - & Spherical & $50-80$ & [155] \\
\hline 1.35 & Nigella arvensis & Antibacterial & - & Spherical & $3-37$ & [156] \\
\hline 1.36 & Punica granatum & Antibacterial & - & Spherical & 5.20 & [157] \\
\hline 1.37 & Pistacia integerrima & Antibacterial & - & Granular & $20-200$ & [158] \\
\hline 1.38 & Plumeria alba & Antibacterial & - & Spherical & $16-28$ & [159] \\
\hline 1.39 & Platycodon grandiflorum & Antimicrobial & - & Spherical & 15 & [160] \\
\hline 1.40 & Rivea hypocrateriformis & Antibacterial & - & Spherical & $10-50$ & [161] \\
\hline 1.41 & Solanum nigrum & Antibacterial & - & Spherical & 50 & [162] \\
\hline 1.42 & Salicornia brachiate & Antibacterial & - & Polydispersed & $22-35$ & [163] \\
\hline 1.43 & Solanum lycopersicums & Antibacterial & - & Diverse & 14 & [164] \\
\hline 1.44 & Trichoderma sp & Antibacterial & - & Pseudospheric & $1-24$ & [165] \\
\hline 1.45 & Trianthema decandra $L$ & Antibacterial & - & $\begin{array}{l}\text { Spherical, hexagonal, } \\
\text { cuboidal }\end{array}$ & $38-80$ & [166] \\
\hline
\end{tabular}


Table 1. Cont.

\begin{tabular}{|c|c|c|c|c|c|c|}
\hline S. No & Name of the Plant & Activity & Cell Line Used & Shape & Size $(\mathrm{nm})$ & Ref. \\
\hline 1.46 & Zingiber officinale (Ginger) & Antibacterial & - & Spherical & $5-15$ & [167] \\
\hline 1.47 & Zizyphus mauritiana & Antibacterial & - & Spherical & $20-40$ & [168] \\
\hline 2 & \multicolumn{6}{|c|}{ Nanoparticles with Anticancer activity } \\
\hline 2.1 & Areca catechu & Anticancer, catalyst & $\mathrm{HeLa}$ & Spherical & 13 & [123] \\
\hline 2.2 & $\begin{array}{c}\text { Artocarpus hirsutus (Wild } \\
\text { jack) }\end{array}$ & Anticancer & $\begin{array}{l}\text { HeLa, RKO } \\
\text { and A549 }\end{array}$ & Spherical & $5-40$ & [169] \\
\hline 2.3 & $\begin{array}{l}\text { Achyranthes Aspera Linn } \\
\text { Seed }\end{array}$ & Anticancer & HeLa (Cervical) & $\begin{array}{l}\text { Spherical, hexagonal, } \\
\text { triangular }\end{array}$ & 9 & [170] \\
\hline 2.4 & Benincasa hispida & Anticancer & HeLa (Cervical) & Spherical & 23 & [126] \\
\hline 2.5 & Brazilian red propolis & Anticancer & $\begin{array}{l}\text { Bladder (T24) and } \\
\text { prostate (PC-3) }\end{array}$ & $\begin{array}{c}\text { Rods, triangular, } \\
\text { pentagonal, hexagonal }\end{array}$ & $8-15$ & [127] \\
\hline 2.6 & Couroupita guianensis & Anticancer & HL-60 & Cubic & 27 & $\begin{array}{l}{[171,} \\
172]\end{array}$ \\
\hline 2.7 & Curcuma wenyujin & Anticancer & $\begin{array}{c}\text { A498 } \\
\text { (renal carcinoma) }\end{array}$ & Spherical & 200 & [173] \\
\hline 2.8 & Ceiba pentandra $(L)$ & Anticancer & $\begin{array}{c}\text { HCT-116 } \\
\text { (colon cancer) }\end{array}$ & Spherical & $20-48$ & [135] \\
\hline 2.9 & Corchorus olitorius & $\begin{array}{c}\text { Antiproliferative } \\
\text { effect }\end{array}$ & $\begin{array}{c}\text { (Breast) MCF-7, } \\
\text { (colon) } \\
\text { HCT-11, and } \\
\text { (hepatocellular) } \\
\text { HepG-2 }\end{array}$ & Triangular, hexagonal & $37-50$ & [174] \\
\hline 2.10 & Diospyros ferrea & Anticancer & $\mathrm{HeLa}$ & Diverse & $70-90$ & [136] \\
\hline 2.11 & Dioscorea batatas & Cytotoxicity & $\begin{array}{c}\text { B16/F10 } \\
\text { (melanoma) }\end{array}$ & Diverse & $19-56$ & [137] \\
\hline 2.12 & Dracocephalum kotschyi & Anticancer & K562 and HeLa & Spherical & 11 & [140] \\
\hline 2.13 & Bay cedar & Anticancer & $\begin{array}{l}\text { Cervical cancer } \\
\quad(\text { HeLa })\end{array}$ & Spherical & $20-25$ & [143] \\
\hline 2.14 & Hevea brasiliensis & $\begin{array}{l}\text { Cytoxicity and } \\
\text { genotoxicity }\end{array}$ & CHO-K1 cells & Spherical, triangular & 50 & [147] \\
\hline 2.15 & Justica wynaadensis & Anticancer & $\begin{array}{l}\text { (Lung cancer) } \\
\text { A549 }\end{array}$ & Spherical & $30-50$ & [148] \\
\hline 2.16 & Jasminum auriculatum & Anticancer & $\begin{array}{l}\text { Cervical cancer } \\
(\text { HeLa })\end{array}$ & Spherical & $8-37$ & [149] \\
\hline 2.17 & Lobila nicotianifolia & Anticancer & $\begin{array}{l}\text { (Lung cancer) } \\
\text { A459 }\end{array}$ & Spherical & 80 & [150] \\
\hline 2.18 & Musa paradisiaca (Banana) & Anticancer & $\begin{array}{l}\text { (Lung cancer) } \\
\text { A459 }\end{array}$ & Diverse & 300 & [154] \\
\hline 2.19 & Marsdenia tenacissima & Anticancer & $\begin{array}{l}\text { (Lung cancer) } \\
\text { A459 }\end{array}$ & Spherical & 50 & [175] \\
\hline 2.20 & Marsilea quadrifolia & Anticancer & $\begin{array}{c}\text { (Lung } \\
\text { adenocarcinoma) } \\
\text { (A549) }\end{array}$ & Spherical & $10-40$ & [176] \\
\hline
\end{tabular}


Table 1. Cont.

\begin{tabular}{|c|c|c|c|c|c|c|}
\hline S. No & Name of the Plant & Activity & Cell Line Used & Shape & Size $(\mathrm{nm})$ & Ref. \\
\hline 2.21 & $\begin{array}{c}\text { Mangifera indica (MI) } \\
\text { mango peel }\end{array}$ & Cytotoxicity & $\begin{array}{l}\text { African green } \\
\text { monkey kidney } \\
\text { normal cells } \\
\text { (CV-1) and fetal } \\
\text { lung fibroblast } \\
\text { cells (WI-38) }\end{array}$ & $\begin{array}{l}\text { Round, triangular, } \\
\text { irregular }\end{array}$ & $19-45$ & [177] \\
\hline 2.22 & Nerium oleander & Anticancer & $\begin{array}{l}\text { MCF-7 (breast } \\
\text { cancer) }\end{array}$ & Spherical & $2-10$ & [178] \\
\hline 2.23 & Nepeta deflersiana & Anticancer & $\begin{array}{c}\text { (Human cervical) } \\
\text { HeLA }\end{array}$ & Cubic & 33 & [179] \\
\hline 2.24 & Nigella arvensis & $\begin{array}{l}\text { Cytotoxicity and } \\
\text { catalytic activities }\end{array}$ & $\begin{array}{c}\text { H1299 and } \\
\text { MCF-7 }\end{array}$ & Spherical & $3-37$ & [156] \\
\hline 2.25 & Orchid & Anticancer & $\begin{array}{c}\text { AMG-13 (breast } \\
\text { cancer) }\end{array}$ & Spherical & $14-50$ & [180] \\
\hline 2.26 & Punica granatum & Anticancer & $\mathrm{HeLa}$ & Spherical & $5-20$ & [157] \\
\hline 2.27 & Korean red ginseng & Anticancer & $\begin{array}{c}\text { (cervical), HeLa, } \\
\text { Hep2 }\end{array}$ & Spherical & $3-40$ & [181] \\
\hline 2.28 & Padina tetrastromatica & Anticancer & $\begin{array}{c}\text { Liver cancer } \\
\text { (HepG2) and } \\
\text { lung cancer } \\
\text { (A549) }\end{array}$ & Spherical & $8-10$ & [182] \\
\hline 2.29 & Scutellaria barbata & Anticancer & $\begin{array}{l}\text { Pancreatic } \\
\text { (PANC-1) }\end{array}$ & Spherical & 154 & [183] \\
\hline 2.30 & saffron stigma (crocin) & Anticancer & $\begin{array}{l}\text { Human breast } \\
\text { cancer cell line } \\
\text { (MCF-7) }\end{array}$ & Spherical & $4-10$ & [184] \\
\hline 2.31 & Sargassum swartzii & Anticancer & $\begin{array}{l}\text { Human cervical } \\
\text { carcinoma (HeLa) }\end{array}$ & Spherical & 35 & [185] \\
\hline 2.32 & Seaweed & Anticancer & $\begin{array}{l}\text { MCF-7 (breast } \\
\text { cancer) }\end{array}$ & Cubic, spherical & $20-50$ & [186] \\
\hline 2.33 & Taxus baccata & Anticancer & $\begin{array}{c}\text { Breast cells } \\
\text { (MCF-7), cervical } \\
\text { cells (HeLa) and } \\
\text { ovarian cells } \\
(\text { Caov-4) }\end{array}$ & Dispersed & 20 & [187] \\
\hline 2.34 & Wedelia trilobata & Anticancer & $\begin{array}{l}\text { HCT } 15 \text { (colon } \\
\text { cancer) }\end{array}$ & Spherical, cubic & $10-50$ & [188] \\
\hline 2.35 & Piper betle & Cytotoxicity & $\begin{array}{l}\text { HeLa and } \\
\text { HEK293 }\end{array}$ & $\begin{array}{l}\text { Prism, cubic, } \\
\text { octahedron, } \\
\text { tetrahedron, } \\
\text { dodecahedron, } \\
\text { triangular }\end{array}$ & $15-55$ & [189] \\
\hline 3 & \multicolumn{6}{|c|}{ Nanoparticles with Antifungal activity } \\
\hline 3.1 & $\begin{array}{c}\text { Abelmoschus } \\
\text { esculentus (Okra) }\end{array}$ & Antifungal & & Crystalline & 62 & [190] \\
\hline 3.2 & $\begin{array}{l}\text { Artemisia vulgaris } \\
\quad \text { (Mugwort) }\end{array}$ & $\begin{array}{c}\text { Larvicidal activity } \\
\text { against Aedes } \\
\text { larvae }\end{array}$ & & $\begin{array}{l}\text { Spherical, triangular, } \\
\text { hexagonal }\end{array}$ & 50-100 & [191] \\
\hline
\end{tabular}


Table 1. Cont.

\begin{tabular}{|c|c|c|c|c|c|c|}
\hline S. No & Name of the Plant & Activity & Cell Line Used & Shape & Size $(\mathrm{nm})$ & Ref. \\
\hline 3.3 & Brazilian red propolis & Antifungal & & $\begin{array}{c}\text { Rods, triangular, } \\
\text { pentagonal, hexagonal }\end{array}$ & $8-15$ & [127] \\
\hline 3.4 & Coreopsis lanceolate & $\begin{array}{c}\text { Detections } \\
\text { of aflatoxins }\end{array}$ & & & & [129] \\
\hline 3.5 & Carthamus tinctorius L & Antifungal & & Triangular, spherical & $40-200$ & [131] \\
\hline 3.6 & Caesalpinia pulcherrima & Antifungal & & Spherical & $10-50$ & [130] \\
\hline 3.7 & Bay cedar & Antifungal & & Spherical & $20-25$ & [143] \\
\hline 3.8 & Helianthus annuus & Antifungal & & Polydispersed & 35 & [146] \\
\hline 3.9 & Nepenthes khasiana & Antifungal & & Spherical & $50-80$ & [155] \\
\hline 3.10 & Punica granatum & Antifungal & & Spherical & $5-20$ & [157] \\
\hline 3.11 & Pistacia integerrima & Antifungal & & Granular & $20-200$ & [158] \\
\hline 3.12 & Rivea hypocrateriformis & Antifungal & & Spherical & $10-50$ & [161] \\
\hline 3.13 & Trianthema decandra $L$ & Antifungal & & $\begin{array}{l}\text { Spherical, hexagonal, } \\
\text { cuboidal }\end{array}$ & $38-80$ & [166] \\
\hline 4 & \multicolumn{6}{|c|}{ Nanoparticles with Antioxidant activity/antidiabetic activity } \\
\hline 4.1 & Areca catechu & $\begin{array}{c}\text { Catalyst, } \\
\text { antioxidant }\end{array}$ & HeLa & Spherical & 13.7 & [123] \\
\hline 4.2 & $\begin{array}{c}\text { Clitoria ternatea (Asian } \\
\text { pigeonwings) }\end{array}$ & Antioxidant & & $\begin{array}{l}\text { Spherical, triangular, } \\
\text { hexagonal }\end{array}$ & 10 & [128] \\
\hline 4.3 & Couroupita guianensis & Antioxidant & HL-60 & Cubic & 27 & $\begin{array}{l}{[171,} \\
172]\end{array}$ \\
\hline 4.4 & Hoveniadulcis & Antioxidant & & Spherical & 20 & [145] \\
\hline 4.5 & Justica wynaadensis & $\begin{array}{l}\text { Antidiabetic and } \\
\text { anti-inflammatory }\end{array}$ & $\begin{array}{c}\text { (Lung cancer) } \\
\text { A549 }\end{array}$ & Spherical & $30-50$ & [148] \\
\hline 4.6 & Nerium oleander & Antioxidant & $\begin{array}{l}\text { MCF-7 (breast } \\
\text { cancer) }\end{array}$ & Spherical & $2-10$ & [178] \\
\hline 4.7 & Nigella arvensis & $\begin{array}{c}\text { Antioxidant, } \\
\text { catalytic activities }\end{array}$ & $\begin{array}{l}\text { H1299 and } \\
\text { MCF-7 }\end{array}$ & Spherical & $3-37$ & [156] \\
\hline
\end{tabular}

It is a known fact that using plants to make gold nanoparticles can result in nanoparticles with distinct biological properties. In a recent study by Mobaraki et al., 2021, using Achillea biebersteinii flower extract, spherical-shaped $(8 \mathrm{~nm})$ gold nanoparticles with anticancer properties against human testicular embryonic carcinoma stem cells were synthesized. The nanoparticles demonstrated dose-dependent cell viability against cancer cells by inducing apoptosis, with half inhibitory concentration (IC50) values of $10 \mathrm{~g} / \mathrm{mL}$ [192]. In another study, Mousavi-Kouhi et al., 2022, synthesized gold nanoparticles from Verbascum speciosum; the green synthesized AuNPs were about $118 \pm 72 \mathrm{~nm}$ in size and very effective against the hepatocellular carcinoma cell line (HepG2) and pathogenic bacteria [193].

Researchers are increasingly interested in the use of naturally occurring materials in biomedicine, and gum tragacanth (GT) has recently shown great promise as a therapeutic substance in tissue engineering and regenerative medicine. GT is a polysaccharide that can be extracted easily from the stems and branches of various Astragalus species. This anionic polymer is biodegradable, non-allergenic, non-toxic, and non-carcinogenic. GT's resistance to microbial, heat, and acid degradation has made it a popular material in industrial (e.g., food packaging) and biomedical applications (e.g., drug delivery). GT has been shown to be a useful reagent in the formation and stabilization of metal nanoparticles over time [194,195]. 


\section{Future Prospective}

When we use green synthesis to make AuNPs, the process is simple. The reaction occurs in a controlled atmosphere with minimal temperature and pressure changes. Their reduction property determines the answer. A plant-based bioactive molecule that functions as a reducing agent usually produces the quickest reaction. Because of the benefits of employing green synthesis, we need to determine which molecules are feasible and to scaleup the commercialization of gold nanoparticles, as well as conduct the research needed for theranostic applications and disease markers. In addition, research should focus on in vivo investigations so that AuNPs can be used further as a medication or carrier for biomedical applications.

\section{Conclusions}

Diverse medicinal plants and their parts are employed to synthesize AuNPs, which have the unique virtue of having anticancer, antibacterial, and antifungal properties with theranostic applications. Nanotheranostics is a rapidly growing research field with enormous potential for improving disease diagnosis and treatment. Green nanoparticle synthesis, with its low capital requirements and operating costs, reduced pollution, and improved biocompatibility and stability, is a new and emerging field with advantages over chemical and physical nanoparticle synthesis methods. The number of biomedical applications in this sector is growing every day, with bioimaging, drug delivery, biosensors, and gene delivery among them. We hope that by focusing the readers' attention on naturally synthesized nanoparticles and their applications, this review will help form a new perspective.

Funding: The authors are grateful to the Deanship of Scientific Research, Najran University, Najran, Saudi Arabia, for funding this research through grant research code NU/RC/MRC/11/1.

Institutional Review Board Statement: Not applicable.

Informed Consent Statement: Not applicable.

Data Availability Statement: Not applicable.

Acknowledgments: The authors are grateful to KLE Technological University, Hubbali, Karnataka, India. The authors sincerely acknowledge the Deanship of Scientific Research, Najran University, Najran, Saudi Arabia, for supporting this research through grant research code NU/RC/MRC/11/1.

Conflicts of Interest: The authors declare no conflict of interest.

\section{References}

1. Zhang, T.; Chen, P.; Sun, Y.; Xing, Y.; Yang, Y.; Dong, Y.; Xu, L.; Yang, Z.; Liu, D. A New Strategy Improves Assembly Efficiency of DNA Mono-Modified Gold Nanoparticles. Chem. Commun. 2011, 47, 5774-5776. [CrossRef] [PubMed]

2. Mukherjee, P.; Bhattacharya, R.; Bone, N.; Lee, Y.K.; Patra, C.R.; Wang, S.; Lu, L.; Secreto, C.; Banerjee, P.C.; Yaszemski, M.J.; et al. Potential Therapeutic Application of Gold Nanoparticles in B-Chronic Lymphocytic Leukemia (BCLL): Enhancing Apoptosis. J. Nanobiotechnology 2007, 5, 4. [CrossRef]

3. Calzolai, L.; Franchini, F.; Gilliland, D.; Rossi, F. Protein-Nanoparticle Interaction: Identification of the Ubiquitin-Gold Nanoparticle Interaction Site. Nano Lett. 2010, 10, 3101-3105. [CrossRef]

4. Jamison, J.A.; Bryant, E.L.; Kadali, S.B.; Wong, M.S.; Colvin, V.L.; Matthews, K.S.; Calabretta, M.K. Altering Protein Surface Charge with Chemical Modification Modulates Protein-Gold Nanoparticle Aggregation. J. Nanopart. Res. 2011, 13, 625-636. [CrossRef]

5. Uehara, N. Polymer-Functionalized Gold Nanoparticles as Versatile Sensing Materials. Anal. Sci. 2010, 26, 1219-1228. [CrossRef] [PubMed]

6. Brown, S.D.; Nativo, P.; Smith, J.-A.; Stirling, D.; Edwards, P.R.; Venugopal, B.; Flint, D.J.; Plumb, J.A.; Graham, D.; Wheate, N.J. Gold Nanoparticles for the Improved Anticancer Drug Delivery of the Active Component of Oxaliplatin. J. Am. Chem. Soc. 2010, 132, 4678-4684. [CrossRef]

7. Khan, J.A.; Kudgus, R.A.; Szabolcs, A.; Dutta, S.; Wang, E.; Cao, S.; Curran, G.L.; Shah, V.; Curley, S.; Mukhopadhyay, D.; et al. Designing Nanoconjugates to Effectively Target Pancreatic Cancer Cells in Vitro and in Vivo. PLoS ONE 2011, 6, e20347. [CrossRef]

8. Singh, P.; Kim, Y.-J.; Zhang, D.; Yang, D.-C. Biological Synthesis of Nanoparticles from Plants and Microorganisms. Trends Biotechnol. 2016, 34, 588-599. [CrossRef]

9. Barry, N.P.E.; Sadler, P.J. Challenges for Metals in Medicine: How Nanotechnology May Help to Shape the Future. ACS Nano 2013, 7, 5654-5659. [CrossRef] 
10. Bakur, A.; Niu, Y.; Kuang, H.; Chen, Q. Synthesis of Gold Nanoparticles Derived from Mannosylerythritol Lipid and Evaluation of Their Bioactivities. AMB Express 2019, 9, 62. [CrossRef]

11. Kumar, R. Anticancer Activity of Eco-Friendly Au Nanoparticles against Lung and Liver Cancer Cells. J. Genet. Eng. Biotechnol. 2016, 14, 195-202.

12. Zhang, Y.; Cui, X.; Shi, F.; Deng, Y. Nano-Gold Catalysis in Fine Chemical Synthesis. Chem. Rev. 2012, 112, 2467-2505. [CrossRef] [PubMed]

13. Duncan, T.V. Applications of Nanotechnology in Food Packaging and Food Safety: Barrier Materials, Antimicrobials and Sensors. J. Colloid Interface Sci. 2011, 363, 1-24. [CrossRef] [PubMed]

14. Dastjerdi, R.; Montazer, M. A Review of the Application of Microbial Nanostructured Materials in the Modification of Textiles Focus on Antimicrobial Properties. Colloids Surf. B Biointerfaces 2010, 79, 5-8. [CrossRef] [PubMed]

15. Mikhailova, E.O. Gold Nanoparticles: Biosynthesis and Potential of Biomedical Application. J. Funct. Biomater. 2021, 12, 70. [CrossRef] [PubMed]

16. Basavegowda, N.; Idhayadhulla, A.; Lee, Y.R. Tyrosinase Inhibitory Activity of Silver Nanoparticles Treated with Hovenia dulcis Fruit Extract: An in Vitro Study. Mater. Lett. 2014, 129, 28-30. [CrossRef]

17. Kulkarni, N.; Muddapur, U. Biosynthesis of Metal Nanoparticles: A Review. J. Nanotechnol. 2014, 2014, 510246. [CrossRef]

18. Lim, Y.Y.; Murtijaya, J. Antioxidant Properties of Phyllanthus amorous Extracts as Affected by Different Drying Methods. LWT-Food Sci. Technol. 2007, 40, 1664-1669. [CrossRef]

19. Sathishkumar, M.; Sneha, K.; Won, S.W.; Cho, C.W.; Kim, S.; Yan, Y.Y. Cinnamomum Zeylanicum Bark Extract and Powder Mediated Green Synthesis of Nano-Crystalline Ag Particles and Its Bacterial Activity. Colloids Surf. B Biointerfaces 2009, 73, 332-338. [CrossRef]

20. Safaepaser, M.; Shahverdi, A.R.; Shahverdi, H.R.; Khorramizadeh, M.R.; Gohari, A.R. Green Synthesis of Small Ag Nanoparticles Using Geraniol and Its Cytotoxicity against Fibrosarcomawehi 164. Avicenna J. Med. Biotechnol. 2009, 1, 111-115.

21. Ju-Nam, Y.; Led, J.R. Manufactured Nanoparticles: An Overview of Their Chemistry, Inclination, and Potential Implication. Sci. Total Environ. 2008, 400, 396-4141. [CrossRef] [PubMed]

22. Kim, K.; Jung, B.; Kim, J.; Kim, W. Effects of Embedding Non-Absorbing Nanoparticles in Organic Photovoltaics on Power Conversion Efficiency. Sol. Energy Mater. Sol. Cells 2010, 94, 1835-1839. [CrossRef]

23. Geoprincy, G.; Srri, B.V.; Poonguzhali, U.; Gandhi, N.N.; Ranganathan, S. A Review on Green Synthesis of Ag Nanoparticles. Asian J. Pharm. Nat. 2013, 6, 8-12.

24. Kruis, F.E.; Fissan, H.; Rellinghaus, B. Sintering and Evaporation Characteristics of Gas-Phase Synthesis of Size-Selected PbS Nanoparticles. Mater. Sci. Eng. B 2000, 69-70, 329-334. [CrossRef]

25. Jung, J.H.; Oh, H.C.; Noh, H.S.; Ji, J.H.; Kim, S.S. Metal Nanoparticle Generation Using a Small Ceramic Heater with a Local Heating Area. J. Aerosol Sci. 2006, 37, 1662-1670. [CrossRef]

26. Iravani, S.; Korbekandi, H.; Mirmohammadi, S.V.; Zolfaghari, B. Synthesis of Ag Nanoparticles; Chemical, Physical and Biological Methods. Res. Pharm. Sci. 2014, 9, 385-406.

27. Tsuji, T.; Iryo, K.; Watanabe, N.; Tsuji, M. Preparation of Ag Nanoparticles by Laser Ablation in Solution: Influence of Laser Wavelength on Particle Size. Appl. Surf. Sci. 2002, 202, 80-85. [CrossRef]

28. Tien, D.-C.; Tseng, K.-H.; Liao, C.-Y.; Huam, J.C.; Tsung, T.T. Discovery of Ionic Ag in Ag Nanoparticles Suspension Fabricated by Arc Discharge Method. J. Alloys Compd. 2008, 463, 408-411. [CrossRef]

29. Iqbal, N.; Iqubal, S.M.S.; Khan, A.A.; Mohammed, T.; Alshabi, A.M.; Aazam, E.S.; Rafiquee, M.Z.A. Effect of CTABr (Surfactant) on the Kinetics of Formation of Silver Nanoparticles by Amla Extract. J. Mol. Liq. 2021, 329, 115537. [CrossRef]

30. Sardar, R.; Shumaker-Parry, J.S. Spectroscopic and Microscopic Investigation of Gold Nanoparticle Formation: Ligand and Temperature Effects on Rate and Particle Size. J. Am. Chem. Soc. 2011, 133, 8179-8190. [CrossRef]

31. Grzelczak, M.; Pérez-Juste, J.; Mulvaney, P.; Liz-Marzán, L.M. Shape Control in Gold Nanoparticle Synthesis. Chem. Soc. Rev. 2008, 37, 1783-1791. [CrossRef] [PubMed]

32. Turkevich, J.; Stevenson, P.C.; Hillier, J. A Study of the Nucleation and Growth Processes in the Synthesis of Colloidal Gold Discuss. Faraday Soc. 1951, 11, 55. [CrossRef]

33. Wilton-Ely, J.D.E.T. The Surface Functionalisation of Gold Nanoparticles with Metal Complexes. Dalton Trans. 2008, 25-29. [CrossRef] [PubMed]

34. Aslan, K.; Pérez-Luna, V.H. Surface Modification of Colloidal Gold by Chemisorption of Alkanethiols in the Presence of a Nonionic Surfactant. Langmuir 2002, 18, 6059-6065. [CrossRef]

35. Lin, S.-Y.; Tsai, Y.-T.; Chen, C.-C.; Lin, C.-M.; Chen, C.-H. Two-Step Functionalization of Neutral and Positively Charged Thiols onto Citrate-Stabilized Au Nanoparticles. J. Phys. Chem. B 2004, 108, 2134-2139. [CrossRef]

36. Brust, M.; Walker, M.; Bethell, D.; Schiffrin, D.J.; Whyman, R. Synthesis of Thiol-Derivatized Gold Nanoparticles in a Two-Phase Liquid-Liquid System. J. Chem. Soc. Chem. Commun. 1994, 801-802. [CrossRef]

37. Hostetler, M.J.; Wingate, J.E.; Zhong, C.-J.; Harris, J.E.; Vachet, R.W.; Clark, M.R.; Londono, J.D.; Green, S.J.; Stokes, J.J.; Wignall, G.D.; et al. Alkanethiolate Gold Cluster Molecules with Core Diameters from 1.5 to 5.2 Nm: Core and Monolayer Properties as a Function of Core Size. Langmuir 1998, 14, 17-30. [CrossRef]

38. Love, J.C.; Estroff, L.A.; Kriebel, J.K.; Nuzzo, R.G.; Whitesides, G.M. Self-Assembled Monolayers of Thiolates on Metals as a Form of Nanotechnology. Chem. Rev. 2005, 105, 1103-1169. [CrossRef] 
39. Noruzi, M.; Zare, D.; Khoshnevisan, K.; Davoodi, D. Rapid Green Synthesis of Gold Nanoparticles Using Rosa Hybrida Petal Extract at Room Temperature. Spectrochim. Acta Part A Mol. Biomol. Spectrosc. 2011, 79, 1461-1465. [CrossRef]

40. Thirumurugan, A.; Jiflin, G.J.; Rajagomathi, G.; Tomy, N.A.; Ramachandran, S.; Jaiganesh, R. Biotechnological synthesis of gold nanoparticles of Azadirachta indica leaf extract. Int. J. Biol. Technol. 2010, 1, 75-77.

41. Herizchi, R.; Abbasi, E.; Milani, M.; Akbarzadeh, A. Current Methods for Synthesis of Gold Nanoparticles. Artif. Cells Nanomed. Biotechnol. 2016, 44, 596-602. [CrossRef] [PubMed]

42. Mohanpuria, P.; Rana, N.K.; Yadav, S.K. Biosynthesis of Nanoparticles: Technological Concepts and Future Applications. J. Nanoparticle Res. 2008, 10, 507-517. [CrossRef]

43. Singh, M.; Kalaivani, R.; Manikandan, S.; Sangeetha, N.; Kumaraguru, A.K. Facile Green Synthesis of Variable Metallic Gold Nanoparticle Using Padina gymnospora, a Brown Marine Macroalga. Appl. Nanosci. 2013, 3, 145-151. [CrossRef]

44. Gardea-Torresdey, J.L.; Parsons, J.G.; Gomez, E.; Peralta-Videa, J.; Troiani, H.E.; Santiago, P.; Yacaman, M.J. Formation and Growth of Au Nanoparticles inside Live Alfalfa Plants. Nano Lett. 2002, 2, 397-401. [CrossRef]

45. Tippayawat, P.; Phromviyo, N.; Boueroy, P.; Chompoosor, A. Green Synthesis of Silver Nanoparticles in Aloe Vera Plant Extract Prepared by a Hydrothermal Method and Their Synergistic Antibacterial Activity. PeerJ 2016, 4, e2589. [CrossRef]

46. Ahmed, S.; Annu; Ikram, S.; Yudha, S.S. Biosynthesis of Gold Nanoparticles: A Green Approach. J. Photochem. Photobiol. B 2016, 161, 141-153. [CrossRef]

47. Narayanan, K.B.; Sakthivel, N. Coriander Leaf Mediated Biosynthesis of Gold Nanoparticles. Mater. Lett. 2008, 62, 4588-4590. [CrossRef]

48. Ankamwar, B. Biosynthesis of Gold Nanoparticles (Green-Gold) Using Leaf Extract Of Terminalia catappa. J. Chem. 2010, 7, 1334-1339. [CrossRef]

49. Smitha, S.L.; Philip, D.; Gopchandran, K.G. Green Synthesis of Gold Nanoparticles Using Cinnamomum zeylanicum Leaf Broth. Spectrochim. Acta Part A Mol. Biomol. Spectrosc. 2009, 74, 735-739. [CrossRef]

50. Parida, U.K.; Bindhani, B.K.; Nayak, P. Green Synthesis and Characterization of Gold Nanoparticles Using Onion (Allium cepa) Extract. World J. Nano Sci. Eng. 2011, 1, 93-98. [CrossRef]

51. Vadlapudi, V.; Kaladhar, D.S.V.G.K. Review: Green Synthesis of Silver and Gold Nanoparticles. Middle-East J. Sci. Res. 2014, 19, 834-842.

52. Arunachalam, K.D.; Annamalai, S.K.; Hari, S. One-Step Green Synthesis and Characterization of Leaf Extract-Mediated Biocompatible Silver and Gold Nanoparticles from Memecylon umbellatum. Int. J. Nanomed. 2013, 8, 1307-1315. [CrossRef] [PubMed]

53. Aromal, S.A.; Vidhu, V.K.; Philip, D. Green Synthesis of Well-Dispersed Gold Nanoparticles Using Macrotyloma uniflorum. Spectrochim. Acta Part A Mol. Biomol. Spectrosc. 2012, 85, 99-104. [CrossRef] [PubMed]

54. Kalishwaralal, K.; Deepak, V.; Ram Kumar Pandian, S.; Kottaisamy, M.; BarathmaniKanth, S.; Kartikeyan, B.; Gurunathan, S. Biosynthesis of Silver and Gold Nanoparticles Using Brevibacterium casei. Colloids Surf. B Biointerfaces 2010, 77, 257-262. [CrossRef]

55. Sujitha, M.V.; Kannan, S. Green Synthesis of Gold Nanoparticles Using Citrus Fruits (Citrus limon, Citrus reticulata and Citrus sinensis) Aqueous Extract and Its Characterization. Spectrochim. Acta Part A Mol. Biomol. Spectrosc. 2013, 102, 15-23. [CrossRef]

56. Tamuly, C.; Hazarika, M.; Borah, S.C.; Das, M.R.; Boruah, M.P. In Situ Biosynthesis of Ag, Au and Bimetallic Nanoparticles Using Piper pedicellatum C.DC: Green Chemistry Approach. Colloids Surf. B Biointerfaces 2013, 102, 627-634. [CrossRef] [PubMed]

57. Kumar, K.M.; Mandal, B.K.; Sinha, M.; Krishnakumar, V. Terminalia chebula Mediated Green and Rapid Synthesis of Gold Nanoparticles. Spectrochim. Acta Part A Mol. Biomol. Spectrosc. 2012, 86, 490-494. [CrossRef]

58. Elavazhagan, T.; Arunachalam, K.D. Memecylon edule Leaf Extract Mediated Green Synthesis of Silver and Gold Nanoparticles. Int. J. Nanomed. 2011, 6, 1265-1278. [CrossRef]

59. Das, R.K.; Gogoi, N.; Bora, U. Green Synthesis of Gold Nanoparticles Using Nyctanthes arbortristis Flower Extract. Bioprocess Biosyst. Eng. 2011, 34, 615-619. [CrossRef]

60. Philip, D.; Unni, C.; Aromal, S.A.; Vidhu, V.K. Murraya koenigii Leaf-Assisted Rapid Green Synthesis of Silver and Gold Nanoparticles. Spectrochim. Acta Part A Mol. Biomol. Spectrosc. 2011, 78, 899-904. [CrossRef]

61. Santhoshkumar, J.; Rajeshkumar, S.; Venkat Kumar, S. Phyto-Assisted Synthesis, Characterization and Applications of Gold Nanoparticles-A Review. Biochem. Biophys. Rep. 2017, 11, 46-57. [CrossRef] [PubMed]

62. Bankar, A.; Joshi, B.; Kumar, A.R.; Zinjarde, S. Banana Peel Extract Mediated Synthesis of Gold Nanoparticles. Colloids Surf. B Biointerfaces 2010, 80, 45-50. [CrossRef]

63. Vinod, V.T.P.; Saravanan, P.; Sreedhar, B.; Devi, D.K.; Sashidhar, R.B. A Facile Synthesis and Characterization of Ag, Au and Pt Nanoparticles Using a Natural Hydrocolloid Gum Kondagogu (Cochlospermum gossypium). Colloids Surf. B Biointerfaces 2011, 83, 291-298. [CrossRef] [PubMed]

64. Kumar, K.P.; Paul, W.; Sharma, C.P. Green Synthesis of Gold Nanoparticles with Zingiber Officinale Extract: Characterization and Blood Compatibility. Process Biochem. 2011, 46, 2007-2013. [CrossRef]

65. Shrikanth, V.M.; Janardhan, B.; Dhananjaya, B.L.; Muddapura, U.M.; More, S.S. Antimicrobial And Antioxidant Activity Of Methanolic Root Extract Of Tabernaemontana alternifolia L. Int. J. Pharm. Pharm. Sci. 2015, 7, 66-69.

66. Majumdar, R.; Bag, B.G.; Ghosh, P. Mimussops Elengi Bark Extract Mediated Green Synthesis of Au Nanoparticles and Study of Its Catalytic Activity. Appl. Nanosci. 2016, 6, 521-528. [CrossRef]

67. Shankar, S.S.; Rai, A.; Ahamed, A.; Sastry, M. Rapid Synthesis of Au, Ag and Bimetallic Au Core Ag Shell Nanoparticles Using Neem (Azadictira indica) Leaf Broth. J. Colloid Interface 2004, 275, 496-502. [CrossRef] 
68. Bakar, A.; Ismail, N.H.H.; Abu Bakar, J. Synthesis and Characterization of Ag Nanoparticles in Natural Rubber. Matter. Chem. Phys. 2007, 104, 276-283. [CrossRef]

69. Chandan, S.P.; Chaudhary, M.; Pasricha, R.; Ahmad, A.; Sastry, M. Synthesis of Au Nano Triangles and Ag Nanoparticles Using Aloevera Plant Extract. Biotechnol. Prog. 2006, 22, 577-583. [CrossRef]

70. Shankar, S.S.; Rai, A.; Ahamed, A.; Sastry, M. Controlling the Optical Properties of Lemongrass Extract Synthesized Au Nano Triangles and Potential Application Infrared-Absorbing Optical Coatings. Chem. Mater. 2005, 17, 566-572. [CrossRef]

71. Kumar, V.; Singh, S.; Srivastava, B.; Bhadouria, R.; Singh, R. Green Synthesis of Silver Nanoparticles Using Leaf Extract of Holoptelea integrifolia and Preliminary Investigation of Its Antioxidant, Anti-Inflammatory, Antidiabetic and Antibacterial Activities. J. Environ. Chem. Eng. 2019, 7, 103094. [CrossRef]

72. Vinosha, M.; Palanisamy, S.; Muthukrishnan, R.; Selvam, S.; Kannapiran, E.; You, S.; Prabhu, N.M. Biogenic Synthesis of Gold Nanoparticles from Halymenia dilatata for Pharmaceutical Applications: Antioxidant, Anti-Cancer and Antibacterial Activities. Process Biochem. 2019, 85, 219-229. [CrossRef]

73. Barai, A.C.; Paul, K.; Dey, A.; Manna, S.; Roy, S.; Bag, B.G.; Mukhopadhyay, C. Green Synthesis of Nerium Oleander-Conjugated Gold Nanoparticles and Study of Its in Vitro Anticancer Activity on MCF-7 Cell Lines and Catalytic Activity. Nano Converg. 2018, 5, 10. [CrossRef] [PubMed]

74. Patil, M.P.; Bayaraa, E.; Subedi, P.; Piad, L.L.A.; Tarte, N.H.; Kim, G.D. Biogenic Synthesis, Characterization of Gold Nanoparticles Using Lonicera japonica and Their Anticancer Activity on HeLa Cells. J. Drug Deliv. Sci. Technol. 2019, 51, 83-90. [CrossRef]

75. Castro-Aceituno, V.; Abbai, R.; Moon, S.S.; Ahn, S.; Mathiyalagan, R.; Kim, Y.-J.; Kim, Y.-J.; Yang, D.C. Pleuropterus Multiflorus (Hasuo) Mediated Straightforward Eco-Friendly Synthesis of Silver, Gold Nanoparticles and Evaluation of Their Anticancer Activity on A549 Lung Cancer Cell Line. Biomed. Pharmacother. 2017, 93, 995-1003. [CrossRef]

76. Arulkumar, S.; Sabesan, M. Biosynthesis and Characterization of Au Nanoparticles Using Anti Parkinsonian Drug Mucuna purines Plant Extract. Int. J. Res. Pharm. Sci. 2010, 1, 417-420.

77. Dash, S.S.; Majumdar, R.; Sikder, A.K.; Bag, B.G.; Patra, B.K. Saraca indica Bark Extract Mediated Green Synthesis of Polyshaped Gold Nanoparticles and Its Application in Catalytic Reduction. Appl. Nanosci. 2014, 4, 485-490. [CrossRef]

78. Wang, C.; Mathiyalagan, R.; Kim, Y.J.; Castro-Aceituno, V.; Singh, P.; Ahn, S.; Wang, D.; Yang, D.C. Rapid Green Synthesis of Silver and Gold Nanoparticles Using Dendropanax morbifera Leaf Extract and Their Anti-Cancer Activities. Int. J. Nanomed. 2016, 10, 3691-3701.

79. Geethalakshmi, R.; Sarada, D.V.L. Gold and Silver Nanoparticles from Trianthema decandra: Synthesis, Characterization, and Antimicrobial Properties. Int. J. Nanomed. 2012, 7, 5375-5384. [CrossRef]

80. Balasubramani, G.; Ramkumar, R.; Krishnaveni, N.; Pazhanimuthu, A.; Natarajan, T.; Sowmiya, R.; Perumal, P. Structural Characterization, Antioxidant and Anticancer Properties of Gold Nanoparticles Synthesized from Leaf Extract(Decoction)of Antigonon leptopus Hook. \& Arn. J. Trace Elements Med. Biol. 2015, 30, 83-89.

81. Raghunandan, D.; Ravishankar, B.; Sharanbasava, G.; Mahesh, D.B.; Harsoor, V.; Yalagatti, M.S.; Bhagawanraju, M.; Venkataraman, A. Anti-Cancer Studies of Noble Metal Nanoparticles Synthesized Using Different Plant Extracts. Cancer Nanotechnol. 2011, 2, 57-65. [CrossRef] [PubMed]

82. Castillo-Henríquez, L.; Alfaro-Aguilar, K.; Ugalde-Álvarez, J.; Vega-Fernández, L.; Montes de Oca-Vásquez, G.; Vega-Baudrit, J.R. Green Synthesis of Gold and Silver Nanoparticles from Plant Extracts and Their Possible Applications as Antimicrobial Agents in the Agricultural Area. Nanomaterials 2020, 10, 1763. [CrossRef] [PubMed]

83. Balwe, S.G.; Rokade, A.A.; Park, S.S.; Jeong, Y.T. Green Synthesis and Characterization of Supported Gold Nanoparticles (Au@PS) from Schisandra Chinensis Fruit Extract: An Efficient and Reusable Catalyst for the Synthesis of Chromeno[2,3-d]Pyrimidin-2Yl)Phenol Derivatives under Solvent-Free Conditions. Catal. Commun. 2019, 128, 105703. [CrossRef]

84. Singh, C.; Baboota, R.K.; Naik, P.K.; Singh, H. Biocompatible Synthesis of Silver and Gold Nanoparticles Using Leaf Extract of Dalbergia sissoo. Adv. Mater. Lett. 2012, 3, 279-285. [CrossRef]

85. Sermakkani, M.; Pandian, T. Biological Synthesis of Ag Nanoparticles Using Medicinal Plant (Cassia italica) Leaves. Int. J. Curr. Res. 2012, 4, 53-58.

86. Konwar Boruah, S.; Kumar Boruah, P.; Sarma, P.; Medhi, C.; Medhi, O.K. Green Synthesis of Gold Nanoparticles Using Camellia sinensis and Kinetics of the Reaction. Adv. Mater. Lett. 2012, 3, 481-486. [CrossRef]

87. Ahmad, M.Z.; Akhter, S.; Rahman, Z.; Akhter, S.; Anwar, M.; Mallik, N.; Ahmad, F.J. Nanometric Gold in Cancer Nanotechnology: Current Status and Future Prospect. J. Pharm. Pharmacol. 2013, 65, 634-651. [CrossRef]

88. Shiva, M.P. Inventory of Forestry Resources for Sustainable Management and Biodiversity Conservation; Indus Publishing: New Delhi, India, 1996.

89. Cowan, M.M. Plant Products as Antimicrobial Agents. Clin. Microbiol. Rev. 1999, 12, 564-582. [CrossRef]

90. Adesokan, A.A.; Yakuba, M.T.; Owoyele, B.V.; Akanji, M.A.; Soladoge, A.; Lawal, O. Effect of Administration of Aqueous and Ethanolic Extracts of Enantia cloranthasteen Bark on Brewer's Yeast Induced Pepsis in Rats. Afr. J. Biochem. Res. 2008, 2, 165-169.

91. Owolabi, M.A.; Coker, H.A.; Jaja, S.I. Flavonoid Metabolites in Urine after Oral Administration of the Aqueous Extract of Persea americana to Rats. J. Nat. Med. 2007, 61, 200-204. [CrossRef]

92. Subramanian, V.; Gautam, V.; Raman, R.; Prahalathan, S.; Ashish, K. The Case of Selected Indian Health Care System; Export-Import Bank of India: Mumbai, India, 2003.

93. John, D.; Britto, A.; Sujin, M.; Dhurmar, M.R. Ethnomedicinal Wisdom of the Manavalakarchi People in Kawgkumari District, Tamilnadu. Int. J. Biol. Technol. 2010, 1, 25-30. 
94. Ullah, S.; Khan, M.R.; Alishah, N.; Shah, S.; Majid, M. Ethno Medical Plant Use-Value in the Lakki Marwat District of Pakistan. J. Ethnopharmacol. 2014, 158, 412-422. [CrossRef] [PubMed]

95. Yuan, H.; Ma, Q.; Ye, L.; Piao, G. The Traditional Medicine and Modern Medicine from Natural Products. Molecules 2016, $21,559$. [CrossRef] [PubMed]

96. Singh, S.P. Himalayan Forest and Ecosystem Services: Incorporating in National Accounting; Central Himalayan Environment Association (CHEA): Nainital, Uttarakhand, India, 2007.

97. Jain, S.K. Medicinal Plants; National Book Trust: Delhi, India, 1968; pp. 1-216.

98. Rajasekharreddy, P.; Rani, P.U.; Sreedhar, B. Qualitative Assessment of Silver and Goldnanoparticle Synthesis in Various Plants: A Photobiological Approach. J. Nanopart. Res. 2010, 12, 1711-1721. [CrossRef]

99. Huang, J.; Li, Q.; Sun, D.; Lu, Y.; Su, Y.; Yang, X.; Wang, H.; Wang, Y.; Shao, W.; He, N.; et al. Biosynthesis of Silver and Gold Nanoparticles by Novel Sundried Cinnamomum camphora Leaf. Nanotechnology 2007, 18, 105104. [CrossRef]

100. Vilchis-Nestor, A.R.; Sánchez-Mendieta, V.; Camacho-López, M.A.; Gómez-Espinosa, R.M.; Camacho-López, M.A.; ArenasAlatorre, J.A. Solvent Less Synthesis and Optical Properties of Au and Ag Nanoparticles Using Camellia sinensis Extract. Mater. Lett. 2008, 62, 3103-3105. [CrossRef]

101. Noruzi, M.; Zare, D.; Davoodi, D. A Rapid Biosynthesis Route for the Preparation of Gold Nanoparticles by Aqueous Extract of Cypress Leaves at Room Temperature. Spectrochim. Acta Part A Mol. Biomol. Spectrosc. 2012, 94, 84-88. [CrossRef]

102. Jiang, X.; Sun, D.; Zhang, G.; He, N.; Liu, H.; Huang, J.; Odoom-Wubah, T.; Li, Q. Investigation of Active Biomolecules Involved in the Nucleation and Growth of Gold Nanoparticles by Artocarpus heterophyllus Lam Leaf Extract. J. Nanopart. Res. 2013, 15, 1741. [CrossRef]

103. Yasmin, A.; Ramesh, K.; Rajeshkumar, S. Optimization and Stabilization of Gold Nanoparticles by Using Herbal Plant Extract with Microwave Heating. Nano Converg. 2014, 1, 12. [CrossRef]

104. Yu, J.; Xu, D.; Guan, H.N.; Wang, C.; Huang, L.K.; Chi, D.F. Facile One-Step Green Synthesis of Gold Nanoparticles Using Citrus maxima Aqueous Extracts and Its Catalytic Activity. Mater. Lett. 2016, 166, 110-112. [CrossRef]

105. Aromal, S.A.; Philip, D. Green Synthesis of Gold Nanoparticles Using Trigonella foenumgraecum and Its Size Dependent Catalytic Activity. Spectrochim. Acta Part A Mol. Biomol. 2012, 97, 1-5. [CrossRef] [PubMed]

106. Annamalai, A.; Babu, S.T.; Jose, N.A.; Sudha, D.; Lyza, C.V. Biosynthesis and characterization of silver and gold nanoparticles using aqueous leaf extraction of Phyllanthus amarus Schum \& Thonn. World Appl. Sci. J. 2011, 13, 1833-1840.

107. Andrei, A.B.; Hassan, Y.; Serban, F. FTIR Spectrophotometric Methods Used for Antioxidant Activity Assay in Medicinal Plants. Appl. Spectrosc. Rev. 2012, 47, 245-255.

108. Bhuvanasree, S.R.; Harini, D.; Rajaram, A.; Rajaram, R. Rapidsyn-thesis of gold nanoparticles with Cissus quadrangularis extract usingmicrowave ir-radiation. Spectrochim. Acta Part A Mol. Biomol. Spectrosc. 2013, 106, 190-196. [CrossRef]

109. Ghodake, G.S.; Deshpande, N.G.; Lee, Y.P.; Jin, E.S. Pear Fruit Extract-Assisted Room-Temperature Biosynthesis of Gold Nanoplates. Colloids Surf. B Biointerfaces 2010, 75, 584-589. [CrossRef]

110. Pasca, R.-D.; Mocanu, A.; Cobzac, S.-C.; Petean, I.; Horovitz, O.; Tomoaia-Cotisel, M. Biogenic Syntheses of Gold Nanoparticles Using Plant Extracts. Part. Sci. Technol. 2014, 32, 131-137. [CrossRef]

111. Jun, S.H.; Kim, H.-S.; Koo, Y.K.; Park, Y.; Kim, J.; Cho, S.; Park, Y. Root Extracts of Polygala tenuifolia for the Green Synthesis of Gold Nanoparticles. J. Nanosci. Nanotechnol. 2014, 14, 6202-6208. [CrossRef]

112. Putnam, C.D.; Hammel, M.; Hura, G.L.; Tainer, J.A. X-Ray Solution Scattering (SAXS.) Combined with Crystallography and Computation: Defining Accurate Macromolecular Structures, Conformations and Assemblies in Solution. Q. Rev. Biophys. 2007, 40, 191-285. [CrossRef]

113. Dubey, S.P.; Lahtinen, M.; Sillanpää, M. Tansy Fruit Mediated Greener Synthesis of Silver and Gold Nanoparticles. Process Biochem. 2010, 45, 1065-1071. [CrossRef]

114. Geng, G.; Chen, P.; Guan, B.; Liu, Y.; Yang, C.; Wang, N.; Liu, M. Sheetlike Gold Nanostructures/Graphene Oxide Composites via a One-Pot Green Fabrication Protocol and Their Interesting Two-Stage Catalytic Behaviors. RSC Adv. 2017, 7, 51838-51846. [CrossRef]

115. Das, P.; Ghosal, K.; Jana, N.K.; Mukherjee, A.; Basak, P. Green Synthesis and Characterization of Silver Nanoparticles Using Belladonna Mother Tincture and Its Efficacy as a Potential Antibacterial and Anti-Inflammatory Agent. Mater. Chem. Phys. 2019, 228, 310-317. [CrossRef]

116. Sozer, N.; Kokini, J.L. Nanotechnology and Its Applications in the Food Sector. Trends Biotechnol. 2009, 27, 82-89. [CrossRef] [PubMed]

117. Cai, W.; Gao, T.; Hong, H.; Sun, J. Applications of Gold Nanoparticles in Cancer Nanotechnology. Nanotechnol. Sci. Appl. 2008, 1, 17-32. [CrossRef] [PubMed]

118. Tang, S.; Hewlett, I. Nanoparticle-Based Immunoassays for Sensitive and Early Detection of HIV-1 Capsid (P24) Antigen. J. Infect. Dis. 2010, 201, S59-S64. [CrossRef]

119. Ayan, K.; Barui, R.; Kotcherlakota, C.-H.R. Medicinal Applications of Metal Nanoparticles Synthesis and Applications in Pharmaceutical Sciences; John Wiley \& Sons: Hoboken, NJ, USA, 2018.

120. Riss, T.L.; Moravec, R.A.; O’Brien, M.A.; Hawkins, E.M.; Niles, A. Homogeneous multiwell assays for measuring cell viability, cytotoxicity, and apoptosis. In Handbook of Assay Development in Drug Discovery; CRC Press: Boca Raton, FL, USA, 2006 ; pp. 385-406. ISBN 9781574444711.

121. Nakkala, J.R.; Mata, R.; Bhagat, E.; Sadras, S.R. Green Synthesis Ofsilver and Gold Nanoparticles from Gymnema Sylvestre Leafextract: Study of Antioxidant and Anti-Cancer Activities. J. Nanopart. Res. 2015, 17, 151. [CrossRef] 
122. Mukherjee, S.; Ghosh, S.; Das, D.K. Gold-Conjugated Green Teananoparticles for Enhanced Anti-Tumor Activities and Hepatoprotection-Synthesis, Characterization and in Vitro Evaluation. J. Nutr. Biochem. 2015, 26, 1283-1297. [CrossRef]

123. Rajan, A.; Vilas, V.; Philip, D. Studies on Catalytic, Antioxidant, Antibacterial and Anti-Cancer Activities of Biogenic Gold Nanoparticles. J. Mol. Liq. 2015, 212, 331-339. [CrossRef]

124. Ganesan, R.; Prabu, H.G. Synthesis of Gold NanoparticlesUsing Herbal Acorus Calamus Rhizome Extract And Coating on Cotton Fabric for Antibacterial and UV Blocking Applications. Arab. J. Chem. 2015, 12, 2166-2174. [CrossRef]

125. Bindhu, M.; Umadevi, M. Antibacterial Activities of Green Synthesized Gold Nanoparticles. Mater. Lett. 2014, 120, 122-125. [CrossRef]

126. Al Saqr, A.; Khafagy, E.-S.; Alalaiwe, A.; Aldawsari, M.F.; Alshahrani, S.M.; Anwer, M.K.; Khan, S.; Lila, A.S.A.; Arab, H.H.; Hegazy, W.A.H. Synthesis of Gold Nanoparticles by Using Green Machinery: Characterization and in Vitro Toxicity. Nanomaterials 2021, 11, 808. [CrossRef]

127. Botteon, C.E.A.; Silva, L.B.; Ccana-Ccapatinta, G.V. Biosynthesis and Characterization of Gold Nanoparticles Using Brazilian Red Propolis and Evaluation of Its Antimicrobial and Anti-Cancer Activities. Sci. Rep. 2021, 11, 1974. [CrossRef]

128. Vanaraj, S.; Jabastin, J.; Sathiskumar, S.; Preethi, K. Production and Characterization of Bio-AuNPs to Induce Synergistic Effect against Multidrug Resistant Bacterial Biofilm. J. Cluster Sci. 2017, 28, 227-244. [CrossRef]

129. Abhijith, K.S.; Thakur, M.S. Application of Green Synthesis of Gold Nanoparticles for Sensitive Detection of Aflatoxin B1 Based on Metal Enhanced Fluorescence. Anal. Methods 2012, 4, 4250-4256. [CrossRef]

130. Nagaraj, B.; Divya, T.; Malakar, B.; Krishnamurthy, N.; Dinesh, R.; Negrila, C.; Ciobanu, C.; Iconaru, S. Phytosynthesis of Gold Nanoparticles Using Caesalpinia pulcherrima (Peacock Flower) Flower Extract And Evaluation of Their Antimicrobial Activities. Dig. J. Nanomater. Biostructures 2012, 7, 899-905.

131. Nagaraj, B.; Malakar, B.; Divya, T.; Krishnamurthy, N.; Liny, P.; Dinesh, R.; Iconaru, S.; Ciobanu, C. Synthesis of Plant Mediated Gold Nanoparticles Using Flower Extracts of Carthamus tinctorius L. (Safflower) and Evaluation Of Their Biological Activities. Dig. J. Nanomater. Biostructures 2012, 7, 1289-1296.

132. Ke, Y.; Al Aboody, M.S.; Alturaiki, W.; Alsagaby, S.A.; Alfaiz, F.A.; Veeraraghavan, V.P.; Mickymaray, S. Photosynthesized Gold Nanoparticles from Catharanthus roseus Induces Caspase-Mediated Apoptosis in Cervical Cancer Cells (HeLa). Artif. Cells Nanomed. Biotechnol. 2019, 47, 1938-1946. [CrossRef]

133. Muthukumar, T.; Sambandam, B.; Aravinthan, A.; Sastry, T.P.; Kim, J.-H. Green Synthesis of Gold Nanoparticlesand Their Enhanced Synergistic Antitumor Activity Using HepG2 and MCF7 Cells and Its Antibacterial Effects. Process Biochem. 2016, 51, 384-391. [CrossRef]

134. Naraginti, S.; Sivakumar, A. Eco-Friendly Synthesis of Silver and Gold Nanoparticles with Enhanced Bactericidal Activity and Study of Silver Catalyzed Reduction of 4-Nitrophenol. Spectrochim. Acta Part A Mol. Biomol. 2014, 128, 357-362. [CrossRef]

135. Brian, M.O.; Selvi, S. Biosynthesis and characterization of gold nanoparticles from Ceiba pentandra (L.) Gaertn bark and evaluation of its antibacterial and anticancer activity. Int. J. Res. Pharm. Sci. 2020, 35, 5643-5650.

136. Ramesh, V.; Armash, A. Green Synthesis of Gold Nanoparticles Against Pathogens and Cancer Cells. IJPR 2015, 10, $250-256$.

137. Sreekanth, T.V.M.; Nagajyothi, P.C.; Supraja, N.; Prasad, T.N.V.K.V. Evaluation of the Antimicrobial Activity and Cytotoxicity of Phytogenic Gold Nanoparticles. Appl. Nanosci. 2015, 5, 595-602. [CrossRef]

138. Khan, A.U.; Yuan, Q.; Wei, Y.; Khan, G.M.; Khan, Z.U.H.; Khan, S.; Ali, F.; Tahir, K.; Ahmad, A.; Khan, F.U. Photocatalytic and Antibacterial Response of Biosynthesized Gold Nanoparticles. J. Photochem. Photobiol. B Biol. 2016, 162, 273-277. [CrossRef] [PubMed]

139. Dorosti, N.; Jamshidi, F. Plant-Mediated Gold Nanoparticles by Dracocephalum kotschyi as Anticholinesterase Agent: Synthesis, Characterisation, and Evaluation of Anticancer and Antibacterial Activity. J. Appl. Biomed. 2016, 14, 235-245. [CrossRef]

140. Annamalai, A.; Christina, V.L.P.; Sudha, D.; Kalpana, M.; Lakshmi, P.T.V. Green Synthesis, Characterization and Antimicrobial Activity of Au NPs Using Euphorbia hirta L. Leaf Extract. Colloids Surf. B Biointerfaces 2013, 108, 60-65. [CrossRef] [PubMed]

141. Gopinath, K.; Kumaraguru, S.; Bhakyaraj, K.; Mohan, S.; Venkatesh, K.S.; Esakkirajan, M.; Kaleeswarran, P.; Alharbi, N.S.; Kadaikunnan, S.; Govindarajan, M. Green Synthesis of Silver, Gold and Silver/Gold Bimetallic Nanoparticles Using the Gloriosa superba Leaf Extract and Their Antibacterial and Antibiofilm Activities. Microb. Pathog. 2016, 101, 1-11. [CrossRef] [PubMed]

142. Abdel-Raouf, N.; Al-Enazi, N.M.; Ibraheem, I.B. Green Biosynthesis of Gold Nanoparticles Using Galaxaura elongata and Characterization of Their Antibacterial Activity. Arab. J. Chem. 2013, 10, S3029-S3039. [CrossRef]

143. Karthika, V.; Arumugam, A.; Gopinath, K.; Kaleeswarran, P.; Govindarajan, M.; Alharbi, N.S.; Kadaikunnan, S.; Khaled, J.M.; Benelli, G. Guazuma ulmifolia Bark-Synthesized Ag, Au and Ag/Au Alloy Nanoparticles: Photocatalytic Potential, DNA/Protein Interactions, Anticancer Activity and Toxicity against 14 Species of Microbial Pathogens. J. Photochem. Photobiol. B Biol. 2017, 167, 189-199. [CrossRef]

144. Bindhu, M.; Rekha, P.V.; Umamaheswari, T.; Umadevi, M. Antibacterial Activities of Hibiscus cannabinus Stemassisted Silver and Gold Nanoparticles. Mater. Lett. 2014, 131, 194-197. [CrossRef]

145. Basavegowda, N.; Idhayadhulla, A.; Lee, Y.R. Phyto-Synthesis of Gold Nanoparticles Using Fruit Extract of Hovenia dulcis and Their Biological Activities. Ind. Crops Prod. 2014, 52, 745-751. [CrossRef]

146. Liny, P.; Divya, T.; Barasa, M.; Nagaraj, B.; Krishnamurthy, N.; Dinesh, R. Preparation of Gold Nanoparticles from Helianthus annuus (Sun Flower) Flowers and Evaluation of Their Antimicrobial Activities. Int. J. Pharma Bio Sci. 2012, 3, $439-446$.

147. Santos, N.M.; Gomes, A.S.; Cavalcante, D.G.; Santos, L.F.; Teixeira, S.R.; Cabrera, F.C.; Job, A.E. Green Synthesis of Colloidal Gold Nanoparticles Using Latex from Hevea brasiliensis and Evaluation of Their in Vitro Cytotoxicity and Genotoxicity. IET Nanobiotechnol. 2019, 13, 307-315. [CrossRef] 
148. Lava, M.B.; Muddapur, U.M.; Basavegowda, N.; More, S.S.; More, V.S. Characterization, anticancer, antibacterial, anti-diabetic and anti-inflammatory activities of green synthesized silver nanoparticles using Justica wynaadensis leaves extract. Mater. Today Proc. 2021, 46, 5942-5947. [CrossRef]

149. Balasubramanian, S.; Kala, S.M.J.; Pushparaj, T.L. Biogenic Synthesis of Gold Nanoparticles Using Jasminum auriculatum Leaf Extract and Their Catalytic, Antimicrobial and Anticancer Activities. J. Drug Deliv. Sci. Technol. 2020, 57, 101620. [CrossRef]

150. Lava, M.B.; Muddapur, U.M.; Nagaraj, B. Synthesis and Characterization of Gold Nanoparticles from Lobelia nicotianifolia Leaf Extract and Its Biological Activities. Adv. Mater. Lett. 2020, 11, 1-4. [CrossRef]

151. Poojary, M.M.; Passamonti, P.; Adhikari, A.V. Green Synthesis of Silver and Gold Nanoparticles Using Root Bark Extract of Mammea suriga: Characterization, Process Optimization, and Their Antibacterial Activity. BioNanoScience 2016, 6, 110-120. [CrossRef]

152. Mubarakali, D.; Thajuddin, N.; Jeganathan, K.; Gunasekaran, M. Plant Extract Mediated Synthesis of Silver and Gold Nanoparticles and Its Antibacterial Activity Against Clinically Isolated Pathogens. Colloids Surf. B Biointerfaces 2011, 85, 360-365. [CrossRef] [PubMed]

153. Ahmad, A.; Syed, F.; Imran, M.; Khan, A.U.; Tahir, K.; Khan, Z.U.H.; Yuan, Q. Phytosynthesis and AntileishmanialActivity of Gold Nanoparticles by Maytenus Royleanus. J. Food Biochem. 2015, 40, 420-427. [CrossRef]

154. Vijayakumar, S.; Vaseeharan, B.; Malaikozhundan, B.; Gopi, N.; Ekambaram, P.; Pachaiappan, R.; Velusamy, P.; Murugan, K.; Benelli, G.; Suresh Kumar, R.; et al. Therapeutic Effects of Gold Nanoparticles Synthesized Using Musa paradisiaca Peel Extract against Multiple Antibiotic Resistant Enterococcus faecalis Biofilms and Human Lung Cancer Cells (A549). Microb. Pathog. 2017, 102, 173-183. [CrossRef]

155. Bhau, B.S.; Ghosh, S.; Puri, S.; Borah, B.; Sarmah, D.K.; Khan, R. Green Synthesis of Gold Nanoparticles from the Leaf Extract of Nepenthes khasiana and Antimicrobial Assay. Adv. Mater. Lett. 2015, 6, 55-58. [CrossRef]

156. Chahardoli, A.; Karimi, N.; Sadeghi, F.; Fattahi, A. Green Approach for Synthesis of Gold Nanoparticles from Nigella arvensis Leaf Extract and Evaluation of Their Antibacterial, Antioxidant, Cytotoxicity and Catalytic Activities. Artif. Cells Nanomed. Biotechnol. 2018, 46, 579-588. [CrossRef] [PubMed]

157. Lokina, S.; Suresh, R.; Giribabu, K.; Stephen, A.; Lakshmi Sundaram, R.; Narayanan, V. Spectroscopic Investigations, Antimicrobial, and Cytotoxic Activity of Green Synthesized Gold Nanoparticles. Spectrochim. Acta Part A Mol. Biomol. Spectrosc. 2014, 129, 484-490. [CrossRef] [PubMed]

158. Islam, N.U.; Jalil, K.; Shahid, M.; Muhammad, N.; Rauf, A. Pistacia integerrima Gall Extract Mediated Green Synthesis of Gold Nanoparticles and Their Biological Activities. Arab. J. Chem. 2019, 12, 2310-2319. [CrossRef]

159. Mata, R.; Bhaskaran, A.; Sadras, S.R. Green-Synthesized Gold Nanoparticles from Plumeria alba Flower Extract to Augment Catalytic Degradation of Organic Dyes and Inhibit Bacterial Growth. Particuology 2016, 24, 78-86. [CrossRef]

160. Anbu, P.; Gopinath, S.C.; Jayanthi, S. Synthesis of Gold Nanoparticles Using Platycodon grandiflorum Extract and Its Antipathogenic Activity under Optimal Conditions. Nanomater. Nanotechnol. 2020, 10, 184798042096169. [CrossRef]

161. Godipurge, S.S.; Yallappa, S.; Biradar, N.J.; Biradar, J.S.; Dhananjaya, B.L.; Hegde, G.; Jagadish, K.; Hegde, G. A Facile and Green Strategy for the Synthesis of $\mathrm{Au}, \mathrm{Ag}$ and $\mathrm{Au}-\mathrm{Ag}$ Alloy Nanoparticles Using Aerial Parts of R. hypocrateriformis Extract and Their Biological Evaluation. Enzyme Microb. Technol. 2016, 95, 174-184. [CrossRef] [PubMed]

162. Muthuvel, A.; Adavallan, K.; Balamurugan, K.; Krishnakumar, N. Biosynthesis of Gold Nanoparticles Using Solanum nigrum Leaf Extract and Screening Their Free Radical Scavenging and Antibacterial Properties. Biomed. Prev. Nutr. 2014, 4, 325-332. [CrossRef]

163. Ahmed, K.B.A.; Subramanian, S.; Sivasubramanian, A.; Veerappan, G.; Veerappan, A. Preparation of Gold Nanoparticles Using Salicornia brachiata Plant Extract and Evaluation of Catalytic and Antibacterial Activity. Spectrochim. Acta Part A Mol. Biomol. Spectrosc. 2014, 130, 54-58. [CrossRef]

164. Bindhu, M.R.; Umadevi, M. Silver and Gold Nanoparticles for Sensor and Antibacterial Applications. Spectrochim. Acta Part A Mol. Biomol. Spectrosc. 2014, 128, 37-45. [CrossRef]

165. Mishra, A.; Kumari, M.; Pandey, S.; Chaudhry, V.; Gupta, K.C.; Nautiyal, C.S. Biocatalytic and Antimicrobial Activities of Gold Nanoparticles Synthesized by Trichoderma sp. Bioresour. Technol. 2014, 166, 235-242. [CrossRef]

166. Geethalakshmi, R.; Sarada, D.V.L. Characterization and Antimicrobial Activity of Gold and Silver Nanoparticles Synthesized Using Saponin Isolated from Trianthema decandra L. Ind. Crops Prod. 2013, 51, 107-115. [CrossRef]

167. Velmurugan, P.; Anbalagan, K.; Manosathyadevan, M.; Lee, K.-J.; Cho, M.; Lee, S.-M.; Park, J.-H.; Oh, S.-G.; Bang, K.-S.; Oh, B.-T. Green Synthesis of Silver and Gold Nanoparticles Using Zingiber officinale Root Extract and Antibacterial Activity of Silver Nanoparticles against Food Pathogens. Bioprocess Biosyst. Eng. 2014, 37, 1935-1943. [CrossRef] [PubMed]

168. Sadeghi, B. Zizyphus mauritiana Extract-Mediated Green and Rapid Synthesis of Gold Nanoparticles and Its Antibacterial Activity. J. Nanostructure Chem. 2015, 5, 265-273. [CrossRef]

169. Vijayashree, I.; Niranjana, P.; Prabhu, G.; Sureshbabu, V.; Manjanna, J. Conjugation of Au Nanoparticles with Chlorambucil for Improved Anti-Cancer Activity. J. Clust. Sci. 2017, 28, 133-148. [CrossRef]

170. Green, R.J. Green Phyto-Synthesis Of Gold Nanoparticles Using Achyranthes aspera Linn Seed-Epicotyls Layer Extracts And Its Anticancer Activity. Asian J. Pharm. Clin. Res. 2014, 7, 136-139.

171. Sathishkumar, G.; Jha, P.K.; Vignesh, V.; Rajkuberan, C.; Jeyaraj, M.; Selvakumar, M.; Jha, R.; Sivaramakrishnan, S. Cannonball Fruit (Couroupita guianensis, Aubl.) Extract Mediated Synthesis of Gold Nanoparticles and Evaluation of Its Antioxidant Activity. J. Mol. Liq. 2016, 215, 229-236. [CrossRef]

172. Geetha, R.; Ashokkumar, T.; Tamilselvan, S. Green Synthesis of Gold Nanoparticles and Their Anti-Cancer Activity. Cancer Nanotechnol. 2013, 4, 91-98. [CrossRef] 
173. Liu, R.; Pei, Q.; Shou, T.; Zhang, W.; Hu, J.; Li, W. Apoptotic Effect of Green Synthesized Gold Nanoparticles from Curcuma wenyujin Extract against Human Renal Cell Carcinoma A498 Cells. Int. J. Nanomed. 2019, 14, 4091-4103. [CrossRef]

174. Ismail, E.H.; Saqer, A.M.A.; Assirey, E.; Naqvi, A.; Okasha, R.M. Successful Green Synthesis of Gold Nanoparticles Using a Corchorus olitorius Extract and Their Antiproliferative Effect in Cancer Cells. Int. J. Mol. Sci. 2018, 19, 2612. [CrossRef]

175. Sun, B.; Hu, N.; Han, L.; Pi, Y.; Gao, Y.; Chen, K. Anticancer Activity of Green Synthesised Gold Nanoparticles from Marsdenia tenacissima Inhibits A549 Cell Proliferation through the Apoptotic Pathway. Artif. Cells Nanomed. Biotechnol. 2019, 47, 4012-4019. [CrossRef]

176. Balashanmugam, P.; Mosachristas, K.; Kowsalya, E. In Vitro Cytotoxicity And Antioxidant Evaluation Of Biogenic Synthesized Gold Nanoparticles From Marsilea quadrifolia On Lung And Ovarian Cancer Cells. Int. J. Appl. Pharm. 2018, 10, $153-158$.

177. Philip, D. Rapid Green Synthesis of Spherical Gold Nanoparticles Using Mangifera indica Leaf. Spectrochim. Acta Part A Mol. Biomol. Spectrosc. 2010, 77, 807-810. [CrossRef] [PubMed]

178. Tahir, K.; Nazir, S.; Li, B.; Khan, A.U.; Khan, Z.U.H.; Gong, P.Y.; Khan, S.U.; Ahmad, A. Nerium oleander Leaves Extract Mediated Synthesis of Gold Nanoparticles and Its Antioxidant Activity. Mater. Lett. 2015, 156, 198-201. [CrossRef]

179. Al-Sheddi, E.S.; Farshori, N.N.; Al-Oqail, M.M.; Al-Massarani, S.M.; Saquib, Q.; Wahab, R.; Musarrat, J.; Al-Khedhairy, A.A.; Siddiqui, M.A. Anticancer Potential of Green Synthesized Silver Nanoparticles Using Extract of Nepeta deflersiana against Human Cervical Cancer Cells (HeLA). Bioinorg. Chem. Appl. 2018, 2018, 9390784. [CrossRef] [PubMed]

180. Yas, R.M.; Ghafoor, A.; Saeed, M.A. Anticancer Effect of Green Synthesized Gold Nanoparticles Using Orchid Extract and Their Characterizations on Breast Cancer AMJ-13 Cell Line. Syst. Rev. Pharm 2021, 12, 500-505.

181. Leonard, K.; Ahmmad, B.; Okamura, H.; Kurawaki, J. In Situ Green Synthesis of Biocompatible Ginseng Capped Gold Nanoparticles with Remarkable Stability. Colloids Surf. B Biointerfaces 2011, 82, 391-396. [CrossRef]

182. Rajeshkumar, S.; Kumar, S.V.; Malarkodi, C.; Vanaja, M.; Paulkumar, K.; Annadurai, G. Optimized Synthesis of Gold Nanoparticles Using Green Chemical Process and Its Invitro Anticancer Activity Against HepG2 and A549 Cell Lines. Mech. Mater. Sci. Eng. J. 2017, 9

183. Wang, L.; Xu, J.; Yan, Y.; Liu, H.; Karunakaran, T.; Li, F. Green Synthesis of Gold Nanoparticles from Scutellaria barbata and Its Anticancer Activity in Pancreatic Cancer Cell (PANC-1). Artif. Cells Nanomed. Biotechnol. 2019, 47, 1617-1627. [CrossRef]

184. Hoshyar, R.; Khayati, G.R.; Poorgholami, M.; Kaykhaii, M. A Novel Green One-Step Synthesis of Gold Nanoparticles Using Crocin and Their Anti-Cancer Activities. J. Photochem. Photobiol. B: Biol. 2016, 159, 237-242. [CrossRef]

185. Dhas, T.S.; Kumar, V.G.; Karthick, V.; Govindaraju, K.; Narayana, T.S. Biosynthesis of Gold Nanoparticles Using Sargassum swartzii and Its Cytotoxicity Effect on HeLa Cells. Spectrochim. Acta Part A Mol. Biomol. Spectrosc. 2014, 133, 102-106. [CrossRef]

186. Namvar, F.; Mohamad, R.; Shameli, K.; Rahman, H.S. Green Synthesis of Gold Nanoparticles (GNP) Using Seaweed Extract and Its Anticancer Effect. Open Conf. Proc. J. 2013, 4, 74. [CrossRef]

187. Kajani, A.A.; Bordbar, A.-K.; Zarkesh Esfahani, S.H.; Razmjou, A. Gold Nanoparticles as Potent Anticancer Agent: Green Synthesis, Characterization, and in Vitro Study. RSC Adv. 2016, 6, 63973-63983. [CrossRef]

188. Dey, A.; Yogamoorthy, S.M. Sundarapandian Green Synthesis of Gold Nanoparticles and Evaluation of Its Cytotoxic Property Against Colon Cancer Cell Line. Pharm. Chem. Sci. 2018, 4, 1-17.

189. Patra, B.; Gautam, R.; Priyadarsini, E.; Rajamani, P.; Pradhan, S.N.; Saravanan, M.; Meena, R. Piper Betle: Augmented Synthesis of Gold Nanoparticles and Its in-Vitro Cytotoxicity Assessment on HeLa and HEK293 Cells. J. Clust. Sci. 2020, 31, 133-145. [CrossRef]

190. Jayaseelan, C.; Ramkumar, R.; Rahuman, A.A.; Perumal, P. Green Synthesis of Gold Nanoparticles Using Seed Aqueous Extract of Abelmoschus esculentus and Its Antifungal Activity. Ind. Crops Prod. 2013, 45, 423-429. [CrossRef]

191. Sundararajan, B.; Kumari, B.R. Novel Synthesis of Gold Nanoparticles Using Artemisia vulgaris L. Leaf Extract and Their Efficacy of Larvicidal Activity against Dengue Fever Vector Aedes aegypti L. J. Trace Elem. Med. Biol. 2017, 43, 187-196. [CrossRef]

192. Mobaraki, F.; Momeni, M.; Taghavizadeh Yazdi, M.E.; Meshkat, Z.; Silanian Toosi, M.; Hosseini, S.M. Plant-Derived Synthesis and Characterization of Gold Nanoparticles: Investigation of Its Antioxidant and Anticancer Activity against Human Testicular Embryonic Carcinoma Stem Cells. Process Biochem. 2021, 111, 167-177. [CrossRef]

193. Mousavi-Kouhi, S.M.; Beyk-Khormizi, A.; Mohammadzadeh, V.; Ashna, M.; Es-haghi, A.; Mashreghi, M.; Hashemzadeh, V.; Mozafarri, H.; Nadaf, M.; Yazdi, M.E.T. Biological Synthesis and Characterization of Gold Nanoparticles Using Verbascum speciosum Schrad. and Cytotoxicity Properties toward HepG2 Cancer Cell Line. Res. Chem. Intermed. 2022, 48, 167-178. [CrossRef]

194. Yazdi, M.T.; Nazarnezhad, S.; Mousavi, S.; Amiri, M.S.; Darroudi, M.; Baino, F.; Kargozar, S. Gum Tragacanth (GT): A Versatile Biocompatible Material beyond Borders. Molecules 2021, 26, 1510. [CrossRef]

195. Akhter, S.; Ahmad, M.Z.; Ahmad, F.J.; Storm, G.; Kok, R.J. Gold Nanoparticles in Theranostic Oncology: Current State of the Art. Expert Opin. Drug Deliv. 2021, 10, 1225-1243. [CrossRef] 